\title{
ISLAMIC BANKS AND FINANCIAL STABILITY: A COMPARATIVE EMPIRICAL ANALYSIS BETWEEN MENA AND SOUTHEAST ASIAN COUNTRIES
}

\author{
Wassim RAJHI ${ }^{*}$, Slim A. HASSAIRI**
}

\begin{abstract}
The aim of this paper is to investigate whether Islamic banks are more stable than conventional banks. To measure the financial stability, we compute the z-score for a sample of banks in 16 countries where Islamic and conventional banks coexist over the period 2000-2008. We use a robust estimation for analyzing data that are contaminated with outliers and leverage points in the data. We use also a quantile estimation to allow us to address the question whether the factors that cause high fragility are systematically different from the factors that cause medium or low fragility. This empirical analysis explores causes of insolvency risk between Middle East and North Africa and South East Asian countries. Finally, by controlling for various factors and by favoring a comparative analysis between the regions, this article is an extension of the study begun by Čihák and Hesse (2010).
\end{abstract}

Key-words - ISLAMIC BANKING, FINANCIAL STABILITY, Z-SCORE, MENA, SOUTHEAST ASIA.

JEL Classification - G21, G32, G33

We gratefully acknowledge valuable comments from Charles Lai-Tong, Catherine Kirby, Laurent Weill and the anonymous referee.

\footnotetext{
*LEAD, Université de Toulon ; Email : wass.rajhi@gmail.com.

** ISCAE, Institut Supérieur de Comptabilité et d'Administration des Entreprises ;

Email : slim.hassairi@univ-amu.fr.
} 


\section{INTRODUCTION}

Emerging financial markets often include Islamic financial services. The International Organization of Securities Commissions predicts that as much as half of the savings of 1.2-1.6 billion Muslims will be in Islamic financial institutions in 2015 (Imam and Kpodar, 2010). These services can be expected to enhance growth in these markets because of the positive relationship between market liquidity and economic growth (Rajan and Zingales, 1998; Levine and Zervos, 1998; Levine, 2005). ${ }^{1}$ Financial development, including emerging Islamic financial services, needs to be orderly and will be adversely affected by adverse macroeconomic or financial shocks. It is therefore interesting to analyse how and why an Islamic banking system might be affected by and respond to these shocks differently from conventional banking systems. This empirical study identifies the key differences between Islamic and conventional banks.

The literature focusing on Islamic finance from the viewpoint of distress and insolvency is not very abundant. A large body of descriptive literature discusses risks in Islamic banking (Sundararajan and Errico, 2002; Grais and $\mathrm{Ku}-$ lathunga, 2006; Grais and Pellegrini, 2006) but does so in theoretical terms, while empirical papers focus on Islamic and conventional banking efficiency (Yudistira, 2004; Hamim, Naziruddin and Syed, 2006; Abdul-Majid, Saal and Battisti, 2010). The role of Islamic banks in financial stability has been analyzed in a consistent, cross-country, empirical fashion in 16 countries between 1993 and 2004 (Čihák and Hesse, 2010). ${ }^{2}$ These authors tried to identify whether market share of Islamic banks has a significant impact on the financial strength of conventional banks, arguing that Islamic financial institutions pose a risk to the financial system that in many regards differs from those posed by the conventional financial system. They used the asset structure (ratio of total loans to total assets) as a proxy for the credit risk. We note the absence of a variable such as a proxy for interest rates risks in their analysis. This article is an extension of the study begun by Čihák and Hesse (2010).

We study the factors determining failures for both conventional and Islamic banks in MENA and in Southeast Asian countries. For each of the regions in our sample, we estimate a separate robust and quantile regression for the period 2000-2008. We show that despite risk management, credit risk measured by the ratio of loan loss provisions to net interest income decreases the z-score in small Islamic banks in MENA countries and large Islamic banks in Southeast Asian countries. The suggested explanation is that Islamic banks in these countries tend to have problems monitoring credit risks. Our results demonstrate that income diversification is a factor of insolvency for small and large Islamic banks in Southeast Asian countries. We find that in Southeast Asian countries, large Islamic and conventional banks cannot coexist in a competitive market without creating crowding out effects. Using the z-score as a measure of bank-

\footnotetext{
${ }^{1}$ It is therefore an opportunity to promote economic growth for Muslims worldwide.

${ }^{2}$ Bahrain, Bangladesh, Brunei, Egypt, Gambia, Indonesia, Jordan, Kuwait, Malaysia, Mauritania,

Pakistan, Qatar, Saudi Arabia, Tunisia, Turkey, United Arab Emirates and Yemen.
} 
specific stability, we find that in general Islamic banks' z-scores are on average higher than those of conventional banks (indicating higher stability than conventional banks). This is not true however for small Islamic banks.

The structure of this paper is as follows. Section 2 discusses our methodology, and introduces the variables and data used in the paper (characterized in more detail in appendix 1). Section 3 presents the empirical results. Section 4 summarizes the conclusions, and suggests topics for further research.

\section{METHODOLOGY AND REVIEW OF LITERATURE}

\subsection{Banking Stability Measure}

We define a measure of the distance-to-default, the z-score, which measures the market value of a bank's assets in relation to the book value of its liabilities. The z-score indicates the distance from insolvency combining accounting measures of profitability, leverage and volatility. ${ }^{3}$ Our primary dependent variable is the $\mathrm{z}$-score as a measure of individual bank risk. The $\mathrm{z}$-score has become a popular measure of bank soundness (Beck, Demirgüç-Kunt and Merrouche, 2010; Čihák and Hesse, 2010). The z-score is inversely related to the probability of a bank's insolvency, i.e., the probability that the value of its assets will become lower than the value of the debt. A higher z-score corresponds to a lower risk of insolvency.

Inspired by Čihák and Hesse (2010), we attempt to establish a relation between z-score and a number of relevant macro and bank-specific indicators. For that purpose and in order to get more insight into insolvency risk developments, we estimate a robust and quantile regression in MENA and Southeast Asian countries. ${ }^{4}$ Because bank size is an important factor in some of the existing papers on bank soundness, we subdivide banks into large and small Islamic and conventional banks. The z-score is calculated by using individual banks. Dependent variable, $\mathrm{z}$-score of bank $i$ at time $t$, is:

$Z_{i, j, t}=\frac{\left(E(R O A A)_{i, j, t}+\frac{e q_{i, j, t}}{t a_{i, j, t}}\right)}{\sigma R O A A_{i, j, t}}$

\footnotetext{
${ }^{3}$ We use the z-score and not a form of probit/logit to see how Islamic banking failure differs from conventional banking failure. It was not the focus of our study given that one typically has only a small number of bank failures and especially for Islamic banks. The z-score measures are being applied at aggregate (sectoral, regional or country) levels, rather than individual bank ones. Strobel (2010) demonstrates that aggregate bank insolvency risk measures that are constructed using the mean of individually calculated z-score measures are inherently biased. The other advantage of using the z-score as a measure of bank stability is that it is based on accounting rather than on market data.

${ }^{4}$ Islamic jurisprudence is based on different schools that may vary from country to country (the Shiah branch and the Sunni branch, which in turn includes the Madhahib, Shafie, Hanafi, Hanbali and Maliki traditions). A typical example is the controversy regarding the Bay al Dayn (debt trading), which is rejected by some Middle East scholars on the grounds that it involves riba (interest). Islam prohibits the trading of debts, unless it is at par value.
} 
where $E(R O A A)_{i, j, t}$ stands for expected return on average assets, $\sigma(R O A A)_{i, j, t}$ denotes standard deviation of return on assets; $(R O A A)$ as a proxy for return volatility; $e q_{i, j, t}$ is bank's equity and reserves; $t a_{i, j, t}$ is bank's assets. The z-score measures the number of standard deviations a return realization has to fall in order to deplete equity, under the assumption of normality of banks' returns.

\subsection{Regression Analysis}

Our approach is mainly to test whether Islamic banks are more or less stable than conventional banks, using regressions of $\mathrm{z}$-scores as a function of a number of variables. We aim to define an insolvency model of the form:

$$
\left(z_{i, j, t}\right)=\alpha+\varphi B_{i, j, t-1}+\gamma I_{j, t-1}+\sum \delta_{S} T_{S}+\sum \varnothing_{S} T_{S} I_{j, t-1}+\sum \varphi_{S} B_{i, j, t-1} T_{S}+\omega M_{j, t-1}+\varepsilon_{i, j, t},
$$

Where the dependent variable is the z-score $\left(Z_{i, j, t}\right)$ for bank $i$ in country $j$ at time $t ; B_{I, J, t-1}$ is a vector of bank-specific variables; $I_{J, t-1}$ contains time-varying industry-specific variables $T_{S}$ and $T_{S} I_{J, t-1}$ are the type of banks and the interaction between the type and some industry-specific variables; $M_{J, t-1}$ denotes macroeconomic variables. Finally, $\varepsilon_{i, j, t}$ is the residual.

\subsection{Control Variables}

The risk of insolvency may be due to factors either internal or external to the bank, such as macro-economic factors, market structures, institutional factors such as regulations and governance indicators (Čihák and Hesse, 2007; 2010). The list of explanatory variables used in regressions incorporates a number of possible risks and bank characteristics discussed in the literature. These are divided into three groups: (i) bank specific variables from bank balance sheets and profit and loss accounts, (ii) national banking sector data and (iii) macroeconomic variables. To distinguish the impact of bank type on the $\mathrm{z}$ score, we include a dummy variable that takes the value of 1 if the bank in question is an Islamic bank and 0 otherwise (i.e., if it is a conventional bank). ${ }^{5}$ In order to control for bank specific characteristics, the regression includes a number of other control variables to separate the impact on financial stability of the Islamic nature of a bank from the impact of other bank-level characteristics, from macroeconomic and other system-level influences.

We include the bank's asset size logged, capital-asset-ratio loans over assets, credit risk, cost-income ratio and liquidity risk. The logarithm of total assets is used as a proxy for bank size, whereas share of loans to assets describes bank asset structure. ${ }^{6}$ As a robustness check, we add the ratio of loan loss provisions to net interest income (credit risk). We expected that a higher ratio would

\footnotetext{
${ }^{5}$ If Islamic banks were relatively weaker than conventional banks, the dummy variable would have a negative sign in the regression explaining z-score.

${ }^{6}$ The asset structure can be interpreted as the profitability potential (as the return on loans is in general higher than on other investments such as government bonds or deposits) as well as the credit risk.
} 
increase the bank insolvency risk. However, this sign might be ambiguous too, as higher loan loss provisions could reflect banks' precautionary reserve building as well as high non-performing loans. Both proxies represent the risk profile of the bank. We interacted a credit risk variable with the Islamic bank dummy because there are differences between Islamic and conventional banks.

For cost efficiency, the cost to income ratio has been used as an indicator of bank cost efficiency. This is a simple measure indicating how well banks manage their total costs (such as overhead expenses) relative to their income; higher values indicate more inefficiency. The cost to income ratio is an efficiency measure similar to operating margin. Unlike the operating margin, lower is better. The cost income ratio is most commonly used in the financial sector. Liquidity risk is measured as a ratio of liquid assets to deposits and short-term funding. ${ }^{7}$ Lack of liquidity can lead to large losses in asset/liability portfolios and it can generate financial distress and insolvency. In general, liquidity reserves promote financial soundness; on the other hand, excess liquidity undermines efficiency and profitability. ${ }^{8}$

Originally developed by Laeven and Levine (2007), income diversity captures the degree to which banks diversify from traditional lending activities (those generating net interest incomes) to other activities. In Islamic banks, the net interest income is generally defined as the sum of the positive and negative income flows associated with the Profit and Loss Sharing (PLS) arrangements. Total operating income of a bank can be classified as interest income and noninterest income (other operating income). Net interest income measures income from traditional interest earning activities. Other operating income measures non-interest earning activities and consists of commissions, fees, as well as trading and asset management income. A higher value of variable income diversity corresponds to a higher degree of diversification. When a bank's income diversity equals one, it fully diversifies its earnings between interest income and non-interest income. When a bank's income diversity equals zero, it is specialized and exclusively engages in either earning interest income or non-interest income. Consequently, an insight into how specific bank characteristics such as levels of revenue diversification interact with bank insolvency risk is of particular interest. We interacted the income diversity variable with the Islamic bank dummy because there are differences between Islamic and conventional banks.

In addition to bank-by-bank data, we incorporate country-specific control variables, using a number of variables that take the same value for all banks in a given year and country. We follow Schaeck and Čihák (2010) by using the inflation rate, the official exchange rate and real gross domestic product growth, since macroeconomic developments are likely to affect the quality of banks assets, as well as the level of bank capitalization. First, we use real GDP growth

\footnotetext{
${ }^{7}$ The choice of a liquidity indicator was constrained, as it was the only uniform indicator available in the BankScope database.

${ }^{8}$ Given their limited access to an interbank market or hedging instruments, Islamic banks are more prone to liquidity risk than conventional banks.
} 
rate where we expect higher growth to reflect better conditions for financial stability. An important variable influencing $\mathrm{z}$-score is the economic activity in the country. Next, we use inflation and official exchange rates, assuming that price and exchange rate stability contribute to the profitability and stability of the banking sector.

We adjust for the impact of the macroeconomic cycle by including LIBOR. ${ }^{9}$ Islamic banks often benchmark the pricing of their instruments to LIBOR. The value of assets such as a deferred sale and lease transaction will vary with the distance between the price at which they were issued and market changes in the benchmark. ${ }^{10}$ LIBOR changes affect an Islamic bank income statement in the same way they do with a conventional bank depending on the share of the balance sheet linked to the benchmark (Chapra and Ahmed, 2002). Finally, interest rate risk measured by 6-month LIBOR was included in the model to control effect on bank stability. We interacted this with the Islamic bank dummy.

Čihák and Hesse (2010) find that the market share of Islamic banks does not have a significant impact on the financial strength of commercial and Islamic banks. Beck, Demirgüç-Kunt and Merrouche (2010) argue that the conventional banks in countries with a higher market share of Islamic banks were less stable. Market share measured as bank assets over total banking sector assets is used as the proxy for market power (Berger, 1995). At the systemic level, we examine the conventional banks' impact on Islamic banks and the hypothesis that the presence of conventional banks lowers systemic stability. For this reason, we have calculated the market share of conventional banks for each year and country and interacted it with the Islamic bank dummy. A negative sign for the interaction of the conventional market share and the Islamic bank dummy would indicate that a higher share of conventional banks reduces their soundness (reduces their z-scores).

We try to capture the possible impact of banking sector concentration on risk-taking behavior by including the Herfindahl-Hirschman index in the model. ${ }^{11}$ Regarding country-specific factors, we include the concentration level,

\footnotetext{
${ }^{9}$ The level of domestic interest rates can also influence banks' risk (Dell' Ariccia and Marquez, 2006; Maddaloni and Peydró, 2011; Borio and Zhu, 2012).

${ }^{10}$ In profit and loss sharing arrangements, the rate of return on financial assets is unknown before to undertaking the transaction. In purchase-resale transactions, a mark-up is determined based on a benchmark rate of return, typically a return determined in international markets such as LIBOR. Islamic financial institutions' balance sheets are exposed to variations of rates of return linked to LIBOR. An increase in LIBOR leads to an increase in the mark-up charged on new transactions and expected returns by investment account holders, compared to the earnings from long-term investments.

11 The Herfindahl-Hirschman index is an economic concept widely applied in competition law, antitrust and technology management. It is the sum of squared market shares (in terms of total assets) of all banks in the country. The index can have values from 0 to 1 , moving from a huge number of banks to a system with only one bank. An increase in this index indicates a decrease in competition and an increase of market power, whereas decreases indicate the opposite.
} 
measured by this index for each country and year, to account for cross-country variation in financial stability caused by differences in market concentration.

Included in the institutions are the process by which governments are selected, monitored and replaced, the capacity of the government to effectively formulate and implement sound policies and the respect of citizens and the state for the institutions that govern economic and social interactions. We expect that banks are more likely to grow in environments where the indicators of governance are built on a solid foundation and might have an effect on banking risk. Furthermore, we controlled for the effect of the institutional environment by using the governance indicators compiled by Kaufmann, Kraay and Mastruzzi (2010). ${ }^{12}$ This indicator captures cross-country differences in institutional developments that might have an effect on banking risks.

All explanatory variables are lagged one year (-1) to capture the possible past effects of these variables on the banks' individual risk. To do that, we estimate the same regressions for different bank sizes between MENA and Southeast Asian countries. We had no a priori assumptions on the sign of coefficients on these variables as they can all affect bank solvency positively or negatively.

To avoid the impact of outliers, we start by a robust estimation for analyzing data that are contaminated with outliers and leverage points in the data. The robust estimation technique assigns lower weights to observations with large residuals, thereby making the estimation less sensitive to outliers, followed by a quantile estimation to allow us to address the question whether the factors that cause high fragility are systematically different from the factors that cause medium or low fragility (Čihák and Hesse, 2007). Several interpretation issues are discussed in detail.

\subsection{The Data}

Our balance sheets and profit and loss accounts data stem from Bureau van Dijk's BankScope database. The BankScope data cover 80-90 percent of the banking systems in terms of total assets. ${ }^{13}$ Our sample covers 467 conventional banks and 90 Islamic banks for the period 2000-2008 in the following 16 countries: 6 Southeast Asian countries and 10 MENA countries (alphabetically ordered): Bahrain, Bangladesh, Brunei, Egypt, Indonesia, Jordan, Kuwait, Malaysia, Pakistan, Qatar, Saudi Arabia, Singapore, Tunisia, Turkey, United Arab

\footnotetext{
${ }^{12}$ The Worldwide Governance Indicators (WGI) project reports governance indicators for 213 economies over the period 1996-2009. Indicators of governance include voice and accountability, political stability, government effectiveness, regulatory quality, the rule of law, and control of corruption.

${ }^{13}$ To decide whether a bank is commercial or Islamic, we have used the BankScope classification as a starting point. BankScope defines Islamic banks as being members of the "International Association of Islamic Banks" plus 20 non-member banks are considered to be "Islamic" by Fitch Ratings. In the BankScope database, some Islamic banks are mistakenly categorized as conventional banks and some as investment banks. The data set does not differentiate between conventional banks with Islamic windows and other conventional banks.
} 
Emirates and Yemen. We have estimated the regressions without Pakistan. It had no significant impact on our main results. We exclude banking systems that are entirely Islamic - Iran and Sudan - from the sample. We have included in conventional banks savings banks and cooperative banks. Some conventional banks have opened dedicated Islamic branches conducting business according to Islamic banking principles. We therefore focus only on the comparison of fully-fledged Islamic banks and conventional banks.

\section{Table 1. Number of Observations by Specialisation and Sub-Samples}

\begin{tabular}{|c|c|c|}
\hline Specialis ation & Observations & Percent \\
\hline \multicolumn{3}{|l|}{ Full Sample } \\
\hline Conventional banks & 2326 & 84.89 \\
\hline Islamic banks & 414 & 15.11 \\
\hline Total & 2740 & 100 \\
\hline \multicolumn{3}{|l|}{ Large Banks } \\
\hline Conventional banks & 1256 & 85.79 \\
\hline Islamic banks & 208 & 14.21 \\
\hline Total & 1464 & 100 \\
\hline \multicolumn{3}{|l|}{ Small Banks } \\
\hline Conventional banks & 1070 & 83.86 \\
\hline Islamic banks & 206 & 16.14 \\
\hline Total & 1276 & 100 \\
\hline
\end{tabular}

Analysis of the sub-samples might show the possible impact of differences in risks of Islamic banks versus conventional banks. Since bank size is an important factor in bank soundness, we subdivide banks into large and small Islamic banks and large and small conventional banks (using total assets of US\$ 1 billion as the cut-off point between small and large banks). The threshold has been used in previous research on small and large banks (Čihák and Hesse, 2007; 2010). In total, we have up to 2740 observations (414 Islamic banks and 2326 conventional banks). Our sample covers conventional banks (84.89\%) and Islamic banks $(15.11 \%)$ as shown in table $1.14 .21 \%$ of the Islamic banks and about $85.79 \%$ conventional banks fall into the large bank category. 16.14 $\%$ of the Islamic banks and $83.86 \%$ of the conventional banks fall into the small bank category.

\section{EMPIRICAL RESULTS}

\subsection{Descriptive Statistics and Pairwise Comparisons}

As a preliminary step in the analysis, we present a comparison of z-scores for various subgroups. Table 2 below illustrates that the treatment of outliers is important. ${ }^{14}$ If the outliers were not included, the result for large conventional

${ }^{14}$ Table 2 includes outliers. 
banks would be reversed (because there are some large conventional banks with extremely high z-scores). In the presence of outliers, ordinary least square estimation can be biased. Because the least squares predictions are dragged towards the outliers, and because the variance of the estimates is artificially inflated, the result is that outliers can be masked. A robust regression is an important tool for analyzing data that are contaminated with outliers and leverage points in the data. A preliminary look at the z-scores suggests high variability in the sample, with a z-score varying from -12.47 to 1614.3 . The average is 25.62 for conventional banks with a maximum of 1614.3. The average for Islamic banks is of 33.61 with a maximum of $1002.7 .^{15}$ Whatever the sample, Islamic banks' $\mathrm{z}$ scores are on average higher than those of conventional banks (indicating higher stability than conventional banks).

Table 2. Descriptive Statistics and T-test Value of Z-score by Specialisation

\begin{tabular}{|c|c|c|c|c|c|c|c|c|c|}
\hline \multirow{3}{*}{$\begin{array}{l}\text { Sample } \\
\text { Specialisation }\end{array}$} & \multicolumn{2}{|c|}{ Full Sample } & $\begin{array}{l}\text { T-test } \\
\text { Value }\end{array}$ & \multicolumn{2}{|c|}{ Large Banks } & $\begin{array}{l}\text { T-test } \\
\text { Value }\end{array}$ & \multicolumn{2}{|c|}{ Small Banks } & \multirow[t]{2}{*}{$\begin{array}{l}\text { T-test } \\
\text { Value }\end{array}$} \\
\hline & \multicolumn{2}{|c|}{ Commercial Islamic } & & \multicolumn{2}{|c|}{ Commercial Islamic } & \multicolumn{3}{|c|}{ Commercial Islamic } & \\
\hline & Banks & Banks & & Banks & Banks & & Banks & Banks & \\
\hline Observations & 2287 & 402 & & 1239 & 204 & & 1048 & 198 & \\
\hline Mean & 25.627 & 33.612 & & 24.418 & 31.586 & & 27.058 & 35.699 & \\
\hline Minimum & -12.475 & -7.691 & & -6.054 & -1.750 & & -12.475 & -7.691 & \\
\hline Maximum & 1614.3 & 1002.7 & & 1107.3 & 208.6 & & 1614.3 & 1002.7 & \\
\hline Kurtosis & 433.445 & 108.223 & & 368.999 & 11.799 & & 368.571 & 73.140 & \\
\hline Skewness & 18.813 & 9.1216 & & 17.112 & 3.344 & & 17.826 & 7.936 & \\
\hline Standard Deviation & 59.988 & 69.415 & & 47.024 & 38.054 & & 72.378 & 91.144 & \\
\hline \multicolumn{10}{|l|}{ Methods of Equality of Means } \\
\hline Pooled (Equal Variance) & & & $(-2.40) * *$ & & & $(2.07)^{* *}$ & & & $(-1.47)$ \\
\hline Satterthwaite (Unequal Variance) & & & $(-2.17)^{* *}$ & & & $(-2.41)^{* *}$ & & & $(-1.26)$ \\
\hline Folded F (Equality of Variances) & & & {$[134] * * *$} & & & {$[1.53] * * *$} & & & {$[1.59] * * *$} \\
\hline
\end{tabular}

F Value []. T Value (). P values: * Significant at 10\%; ** significant at 5\%; *** significant at $1 \%$

The statistical tests in the table 2 indicate whether the mean for z-score is the same for conventional and Islamic banks. The statistical test for the equality of means is reported for both equal and unequal variances. Both tests indicate evidence of a significant difference between z-scores, except for small Islamic banks (for the pooled and for the Satterthwaite tests). These tests assume that the observations are normally distributed. The equality of variances test does indicate a significant difference in the variances. The results indicate that there is a statistically significant difference between the mean z-score for conventional and Islamic banks. In other words, Islamic banks have a statistically signifi-

\footnotetext{
15 These comparisons of $z$-scores between banks are useful, but may overlook some additional factors that explain bank-to-bank variation in z-scores. We will therefore examine this issue more formally using robust and quantile regression analysis.
} 
cant higher mean than conventional banks, except for small Islamic banks. Our results are different from those found by Čihák and Hesse (2010). ${ }^{16}$

\subsection{Regression Analysis}

To separate the financial stability impact of the Islamic nature of a bank from the impact of other bank-level characteristics, and from macroeconomic and other system-level influences, we turn to regression analysis, following the methodology described in section 1. We run several specifications between MENA and Southeast Asian countries. The results for the robust estimation technique, which assigns lower weights to observations with large residuals, thereby making the estimation less sensitive to outliers, are shown in the tables 3 to 11 , while table 12 shows results of quantile estimation technique.

The regressions do not confirm the result from the comparison of z-scores in table 2. We find that large Islamic banks tend to be more stable than large conventional banks, while small Islamic banks tend to be less stable than small conventional banks. The sign of the Islamic dummy variable is predominantly positive in Southeast Asian countries, significant in the regressions for large banks at the 1 percent level in the specifications (1) (2) and (4). The regressions show that in MENA countries small Islamic banks are less stable; the sign of the Islamic dummy variable is negative at the 1 percent level in the specifications (3) and (4). Nevertheless, in Southeast Asian countries, the results for small Islamic banks become less clear-cut, reflecting the opposite signs in the specifications (1) and (2).

The bank's asset size logged is on average positively related to bank stability at the 1 and 10 percent levels in the full sample and large banks in MENA and Southeast Asian countries. The slope coefficient is predominantly positive at the 1 and 5 percent levels; except for small banks in MENA and Southeast Asian countries, where the results are negative at the 1 and 5 percent levels. The bank's asset size logged is on average negatively related to bank stability for small banks.

Whatever the sample, our econometric results indicate that a higher costto-income ratio has a consistently negative link to the z-scores in all specifications at the 10, 5 and 1 percent levels. Our results show that higher liquidity increases bank stability; the results are significant at the 1,5 and 10 percent levels. The impact of the liquidity risk does explain much of the variation in $\mathrm{z}$ scores, so we can clearly drawn conclusions about the association between liquidity and stability.

\footnotetext{
${ }^{16}$ Their results indicate that: (i) small Islamic banks tend to be financially stronger than small conventional banks, (ii) large conventional banks tend to be financially stronger than large Islamic banks and (iii) small Islamic banks tend to be financially stronger than large Islamic banks.
} 
Table 3. Robust Estimation (Full Sample - All Countries) Least Trimmed Squares Parameter - Dependent Variable: Z-score

\begin{tabular}{|c|c|c|c|c|}
\hline \multirow{2}{*}{$\begin{array}{l}\text { Region/Country } \\
\text { Estimate no. }\end{array}$} & \multicolumn{4}{|c|}{ All Countries } \\
\hline & (1) & (2) & (3) & (4) \\
\hline & 1.6303 & 2.4049 & 17.1106 & 2.4980 \\
\hline Islamic Bank Dummy & $(0.3002)$ & $(\mathbf{0 . 1 3 4 5 )}$ & $(0.0048) * * *$ & $(0.7054)$ \\
\hline & 0.9413 & 0.8909 & 0.2303 & 0.7884 \\
\hline Log Asset (-1) & $(<.0001)^{* * * *}$ & $(<.0001)^{* * * *}$ & $(0.2604)$ & $(<.0001)^{* * *}$ \\
\hline & 0.0543 & 0.0437 & 0.0317 & 0.0300 \\
\hline Loans/ Assets (-1) & $(0.0012) * * *$ & $(0.0107)^{* *}$ & $(0.0817)^{*}$ & $(0.0982)^{*}$ \\
\hline Credit Risk (-1) & $\begin{array}{c}-0.0008 \\
(0.3800)\end{array}$ & $\begin{array}{l}-0.0009 \\
(0.3331)\end{array}$ & $\begin{array}{c}0.0009 \\
(0.3882)\end{array}$ & $\begin{array}{l}-0.0015 \\
(0.1362)\end{array}$ \\
\hline Credit Risk * Islamic Bank Dummy & -0.0001 & 0.0000 & -0.0269 & 0.0010 \\
\hline$(-1)$ & $(0.9703)$ & $(0.9793)$ & $(0.1037)$ & $(0.5937)$ \\
\hline & -0.1290 & -0.1386 & -0.0770 & -0.8754 \\
\hline Income Diversity $(-1)$ & $(0.5314)$ & $(0.4970)$ & $(0.7070)$ & $(0.0054)^{* * *}$ \\
\hline $\begin{array}{l}\text { Income Diversity * Islamic Dummy } \\
(-1)\end{array}$ & $\begin{array}{l}1.4184 \\
(0.5512)\end{array}$ & $\begin{array}{l}1.0468 \\
(0.6571)\end{array}$ & $\begin{array}{l}1.7899 \\
(0.4608)\end{array}$ & $\begin{array}{c}2.2368 \\
(0.4441)\end{array}$ \\
\hline & -0.0917 & -0.0920 & -0.0948 & -0.1101 \\
\hline Cost / Income Ratio (-1) & $(<.0001)^{* * * *}$ & $(<.0001) * * *$ & $(<.0001) * * *$ & $(<.0001) * * *$ \\
\hline $\begin{array}{l}\text { Liquid Assets Customer / Short Term Fund } \\
(-1)\end{array}$ & $\begin{array}{c}0.0653 \\
(<.0001)^{* * *}\end{array}$ & $\begin{array}{c}0.0663 \\
(<.0001)^{* * *}\end{array}$ & $\begin{array}{c}0.0552 \\
(<.0001)^{* * *}\end{array}$ & $\begin{array}{l}0.0702 \\
(<.0001)^{* * *}\end{array}$ \\
\hline & & -8.4326 & -18.3769 & -10.0749 \\
\hline Herfindahl-Hirschman Index (-1) & & $(0.0161)^{* *}$ & $(<.0001)^{* * * *}$ & $(0.0051)^{* * *}$ \\
\hline & & 0.0187 & 0.0819 & 0.0386 \\
\hline Market Share Conventional Banks (- 1) & & $(0.5333)$ & $(0.0224)^{* *}$ & $(0.2758)$ \\
\hline Market Share Conventional Banks*Islamic & & & -0.1829 & -0.0187 \\
\hline Bank Dummy (-1) & & & $\begin{array}{l}(0.0081) * * * \\
5.1854\end{array}$ & $(0.7926)$ \\
\hline Governance & & & $(<.0001)^{* * * *}$ & \\
\hline LIBOR $(-1)$ & & & & $\begin{array}{c}0.1175 \\
(0.4894)\end{array}$ \\
\hline $\begin{array}{l}\text { LIBOR* Islamic Bank Dummy } \\
(-1)\end{array}$ & & & & $\begin{array}{c}\mathbf{0 . 1 5 4 2} \\
(\mathbf{0 . 7 8 8 6}) \\
0.1960\end{array}$ \\
\hline Real GDP Growth $(-1)$ & & & & $\begin{array}{l}(0.0952)^{*} \\
-0.1154\end{array}$ \\
\hline Inflation $(-1)$ & & & & $(0.0063)^{* * * *}$ \\
\hline Official Exchange Rate (-1) & & & & $(<.0001) * * *$ \\
\hline & 4.3001 & 4.8679 & 12.5860 & 6.5026 \\
\hline Constant & $(0.1372)$ & $(0.2279)$ & $(0.0092) * * *$ & $(0.1527)$ \\
\hline Observations & 2059 & 2059 & 1856 & 1924 \\
\hline R-Squared & 0.1456 & 0.1509 & 0.1652 & 0.1841 \\
\hline
\end{tabular}

Robust $P$ values in parentheses $*$ Significant at 10\%; ** significant at 5\%; *** significant at $1 \%$.

Estimates of regression coefficients are reported in the following model: $\left(z_{i, j, t}\right)=\alpha+\varphi B_{i, j, t-1}+\gamma I_{j, t-1}+\sum \delta_{S} T_{S}+\sum \emptyset_{S} T_{S} I_{j, t-1}+\sum \varphi_{S} B_{i, j, t-1} T_{S}+\omega M_{j, t-1}+\varepsilon_{i, j, t}$. This table reports the regression results for the robust estimation with sample splits between MENA and Southeast Asia countries. The dependent variable is the z-score, a high index means a low bank insolvency risk exposure; thus the relationship between independent variables and bank insolvency risk exposure is reversed from the sign in this table. If Islamic banks were relatively weaker than conventional banks, the dummy variable would have a negative sign in the regression explaining z-scores. A negative sign for the interaction of independent variables and the Islamic bank dummy would indicate that a higher value reduces their soundness (reduces their $z$ scores). 
Table 4. Robust Estimation (Full Sample - Middle East and North Africa) Least Trimmed Squares Parameter - Dependent Variable: Z-score

\begin{tabular}{|c|c|c|c|c|}
\hline \multirow{2}{*}{$\begin{array}{l}\text { Region/Country } \\
\text { Estimate no. }\end{array}$} & \multicolumn{4}{|c|}{ Middle East and North Africa } \\
\hline & (1) & (2) & (3) & (4) \\
\hline \multirow{3}{*}{ Islamic Bank Dummy } & -0.2882 & 0.9799 & -6.8376 & -18.1131 \\
\hline & $(0.8505)$ & $(0.5189)$ & $(0.2701)$ & $(0.0048)^{* * *}$ \\
\hline & 1.1800 & 1.2074 & 1.1314 & 1.0764 \\
\hline \multirow[t]{2}{*}{ Log Asset (-1) } & $(<.0001) * * *$ & $(<.0001)^{* * * *}$ & $(<.0001)^{* * * *}$ & $(<.0001)^{* * * *}$ \\
\hline & 0.0712 & 0.0606 & 0.0651 & 0.0509 \\
\hline \multirow[t]{2}{*}{ Loans/ Assets (-1) } & $(0.0004) * * *$ & $(0.0036)^{* * * *}$ & $(0.0054)^{* * *}$ & $(0.0235) * *$ \\
\hline & $\begin{array}{c}-0.0068 \\
(0.0179)^{* *}\end{array}$ & $\begin{array}{c}-0.0068 \\
(0.0183) * *\end{array}$ & $\begin{array}{c}-0.0058 \\
(0.0483)^{* *}\end{array}$ & $\begin{array}{c}-0.0102 \\
(0.0029) * * *\end{array}$ \\
\hline \multirow{3}{*}{$\begin{array}{l}\text { Credit Risk * Islamic Dummy } \\
(-1)\end{array}$} & 0.0065 & -0.0171 & -0.0138 & 0.0006 \\
\hline & $(0.0379)^{* *}$ & $(0.2309)$ & $(\mathbf{0 . 3 3 8 2})$ & $(0.9720)$ \\
\hline & -0.0442 & -0.0482 & -0.4109 & -0.5798 \\
\hline Income Diversity $(-1)$ & $(0.5079)$ & $(0.4685)$ & $(0.1517)$ & $(0.0385) * *$ \\
\hline \multirow[t]{2}{*}{$\begin{array}{l}\text { Income Diversity * Islamic Bank Dummy } \\
(-1)\end{array}$} & $\begin{array}{c}7.7296 \\
(0.0004)^{* * *}\end{array}$ & $\begin{array}{c}6.2538 \\
(0.0039)^{* * *}\end{array}$ & $\begin{array}{c}5.6403 \\
(0.0110) * *\end{array}$ & $\begin{array}{c}12.3567 \\
(<.0001)^{* * * *}\end{array}$ \\
\hline & -0.1141 & -0.1104 & -0.0945 & -0.0750 \\
\hline Cost / Income Ratio (-1) & $(<.0001)^{* * *}$ & $(<.0001) * * *$ & $(<.0001) * * *$ & $(<.0001) * * *$ \\
\hline \multirow{2}{*}{$\begin{array}{l}\text { Liquid Assets Customer / Short Term Fund } \\
(-1)\end{array}$} & 0.0630 & 0.0667 & 0.0361 & 0.0830 \\
\hline & $(<.0001)^{* * *}$ & $(<.0001)^{* * *}$ & $(<.0001)^{* * *}$ & $(<.0001)^{* * *}$ \\
\hline \multirow{2}{*}{ Herfindahl-Hirschman Index (-1) } & & $\begin{array}{l}-5.2253 \\
(0.2504)\end{array}$ & $\begin{array}{l}-4.2052 \\
(0.4007)\end{array}$ & -4.3887 \\
\hline & & -0.0371 & -0.0787 & -0.0653 \\
\hline \multirow{3}{*}{$\begin{array}{l}\text { Market Share Conventional Banks }(-1) \\
\text { Market Share Conventional Banks*Islamic } \\
\text { Bank Dummy (- 1) }\end{array}$} & & $(0.2624)$ & $(0.0492)^{* *}$ & $(0.0980) *$ \\
\hline & & & 0.1210 & 0.1270 \\
\hline & & & & $(0.0665)^{*}$ \\
\hline Governance & & & $(0.0733)^{*}$ & \\
\hline LIBOR (-1) & & & & $\begin{array}{c}-0.1379 \\
(0.5240)\end{array}$ \\
\hline \multirow[t]{2}{*}{$\begin{array}{l}\text { LIBOR* Islamic Bank Dummy } \\
(-1)\end{array}$} & & & & $\begin{array}{c}1.2114 \\
(0.0272)^{* *}\end{array}$ \\
\hline & & & & 0.1170 \\
\hline \multirow[t]{2}{*}{ Real GDP Growth (-1) } & & & & $(0.3008)$ \\
\hline & & & & $\begin{array}{l}-0.0591 \\
(0.1466)\end{array}$ \\
\hline Inflation (-1) & & & & -0.0469 \\
\hline \multirow{2}{*}{ Official Exchange Rate (-1) } & & & & $(0.1222)$ \\
\hline & -1.2870 & 2.8543 & 5.5769 & 8.4458 \\
\hline Constant & $(0.7373)$ & $(0.5123)$ & $(0.2775)$ & $(0.0762)^{*}$ \\
\hline Observations & 1024 & 1024 & 921 & 891 \\
\hline R-Squared & 0.2047 & 0.2330 & 0.2378 & 0.3161 \\
\hline
\end{tabular}

Robust $P$ values in parentheses $*$ Significant at $10 \%$;* significant at $5 \%$; *** significant at $1 \%$. 
Table 5. Robust Estimation (Full Sample - Southeast Asia and Pakistan) Least Trimmed Squares Parameter - Dependent Variable: Z-score

\begin{tabular}{|c|c|c|c|c|}
\hline \multirow{2}{*}{$\begin{array}{l}\text { Region/Country } \\
\text { Estimate no. }\end{array}$} & \multicolumn{4}{|c|}{ Southeast Asia and Pakistan } \\
\hline & (1) & (2) & (3) & (4) \\
\hline & 27.0732 & 27.2524 & 64.4143 & 65.8398 \\
\hline Islamic Bank Dummy & $(<.0001) * * *$ & $(<.0001) * * *$ & $(<.0001)^{* * * *}$ & $(0.0003)^{* * *}$ \\
\hline & 1.1848 & 1.0209 & -0.9108 & 0.6976 \\
\hline Log Asset (-1) & $(<.0001) * * *$ & $(0.0001)^{* * * *}$ & $(0.0037)^{* * *}$ & $(0.0118) * *$ \\
\hline & 0.0085 & 0.0083 & -0.0093 & 0.0066 \\
\hline Loans/ Assets (-1) & $(0.7455)$ & $(0.7561)$ & $(0.7247)$ & $(0.8025)$ \\
\hline & 0.0001 & 0.0001 & 0.0001 & 0.0001 \\
\hline Credit Risk (- 1) & $(0.8852)$ & $(0.9211)$ & $(0.9544)$ & $(0.9384)$ \\
\hline $\begin{array}{l}\text { Credit Risk * Islamic Bank Dummy } \\
(-1)\end{array}$ & $\begin{array}{l}-0.2765 \\
(0.0431)^{*} *\end{array}$ & $\begin{array}{c}-0.2658 \\
(0.0502) *\end{array}$ & $\begin{array}{c}-0.3544 \\
(0.0071) * * *\end{array}$ & $\begin{array}{c}-0.1842 \\
(0.1872)\end{array}$ \\
\hline & -3.5375 & -3.7024 & -4.7155 & -3.8694 \\
\hline Income Diversity $(-1)$ & $(0.0003)^{* * *}$ & $(0.0001)^{* * *}$ & $(<.0001)^{* * *}$ & $(<.0001) * * *$ \\
\hline $\begin{array}{l}\text { Income Diversity * Islamic Bank Dummy } \\
(-1)\end{array}$ & $\begin{array}{c}-40.1238 \\
(<.0001)^{* * *}\end{array}$ & $\frac{-40.7372}{(<.0001)^{* * *}}$ & $(0.0021)^{* * *}$ & $\begin{array}{c}-31.2994 \\
(<.0001) * * *\end{array}$ \\
\hline & -0.0754 & -0.0691 & -0.0850 & -0.0621 \\
\hline Cost / Income Ratio (-1) & $(<.0001)^{* * *}$ & $(<.0001) * * *$ & $(<.0001)^{* * *}$ & $(<.0001) * * *$ \\
\hline Liquid Assets Customer / Short Term Fund & 0.1360 & 0.1411 & 0.1403 & 0.0448 \\
\hline$(-1)$ & $(<.0001)^{* * *}$ & $(<.0001)^{* * *}$ & $(<.0001) * * *$ & $(<.0001)^{* * * *}$ \\
\hline Herfindahl-Hirschman Index $(-1)$ & & $\begin{array}{l}-5.4796 \\
(0.3614)\end{array}$ & $\begin{array}{l}-1.6679 \\
(0.8431)\end{array}$ & $\begin{array}{l}-7.6589 \\
(0.2097)\end{array}$ \\
\hline & & -0.0036 & 0.4005 & 0.2414 \\
\hline Market Share Conventional Banks (- 1) & & $(0.9624)$ & $(<.0001)^{* * *}$ & $(0.0106)^{* *}$ \\
\hline Market Share Conventional Banks*Islamic & & & -0.4876 & -0.3317 \\
\hline Bank Dummy (- 1) & & & & (0.1020) \\
\hline Governance & & & $(<.0001)^{* * *}$ & \\
\hline J IBOR (1) & & & & 0.3013 \\
\hline LIBOR* Islamic Bank Dummy & & & & $\begin{array}{l}(0.23 / 4) \\
-3.4249\end{array}$ \\
\hline$(-1)$ & & & & $\begin{array}{c}(\mathbf{0 . 0 3 0 7})^{* *} \\
0.5922\end{array}$ \\
\hline Real GDP Growth (-1) & & & & $(0.0482) * *$ \\
\hline Inflation $(-1)$ & & & & $(<.0001)^{* * *}$ \\
\hline & & & & -0.3368 \\
\hline Official Exchange Rate (-1) & & & & $(<.0001) * * *$ \\
\hline & 4.9788 & 8.3199 & 5.1899 & -11.2119 \\
\hline Constant & $(0.2645)$ & $(0.3787)$ & $(0.6392)$ & $(0.2955)$ \\
\hline Observations & 1035 & 1035 & 935 & 1033 \\
\hline R-Squared & 0.1979 & 0.2075 & 0.3218 & 0.2453 \\
\hline
\end{tabular}

Robust $P$ values in parentheses $*$ Significant at $10 \%$; * significant at $5 \%$; ** significant at $1 \%$. 
Table 6. Robust Estimation (Large Banks - All Countries) Least Trimmed Squares Parameter - Dependent Variable: Z-score

\begin{tabular}{|c|c|c|c|c|}
\hline \multirow{2}{*}{$\begin{array}{l}\text { Region/Country } \\
\text { Estimate no. }\end{array}$} & \multicolumn{4}{|c|}{ All Countries } \\
\hline & (1) & (2) & (3) & (4) \\
\hline \multirow{3}{*}{ Islamic Bank Dummy } & 6.4977 & 7.2694 & 16.0866 & 24.1546 \\
\hline & $(0.0009) * * *$ & $(0.0005) * * *$ & $(0.1596)$ & $(0.0373)^{* *}$ \\
\hline & 3.3443 & 3.4005 & 2.5492 & 3.5719 \\
\hline \multirow[t]{2}{*}{ Log Asset (-1) } & $(<.0001)^{* * * *}$ & $(<.0001)^{* * * *}$ & $(<.0001)^{* * *}$ & $(<.0001)^{* * *}$ \\
\hline & 0.1110 & 0.1273 & 0.0454 & 0.1382 \\
\hline \multirow{2}{*}{ Loans/ Assets (-1) } & $(<.0001)^{* * *}$ & $(<.0001)^{* * * *}$ & $(0.1090)$ & $(<.0001)^{* * *}$ \\
\hline & 0.0009 & 0.0011 & 0.0013 & 0.0008 \\
\hline Credit Risk (- 1) & $(0.4812)$ & $(0.4023)$ & $(0.2770)$ & $(0.5656)$ \\
\hline \multirow[t]{2}{*}{$\begin{array}{l}\text { Credit Risk * Islamic Bank Dummy } \\
(-1)\end{array}$} & $\begin{array}{l}0.0189 \\
(0.6597)\end{array}$ & $\begin{array}{l}0.0050 \\
(0.9092)\end{array}$ & $\begin{array}{c}0.0727 \\
(\mathbf{0 . 1 0 7 5})\end{array}$ & $\begin{array}{l}0.0370 \\
(0.4496)\end{array}$ \\
\hline & 1.5793 & 1.4175 & -0.0213 & $\begin{array}{l}(0.4+40) \\
-0.8662\end{array}$ \\
\hline Income Diversity $(-1)$ & $(0.1954)$ & $(0.2500)$ & $(0.7810)$ & $(0.3695)$ \\
\hline \multirow{3}{*}{$\begin{array}{l}\text { Income Diversity * Islamic Dummy } \\
(-1)\end{array}$} & -4.8437 & -4.8741 & 1.6256 & -3.5978 \\
\hline & $(0.1069)$ & $(0.1096)$ & $(0.5567)$ & $(0.3105)$ \\
\hline & -0.1059 & -0.1088 & -0.0982 & -0.1404 \\
\hline Cost / Income Ratio (-1) & $(<.0001)^{* * *}$ & $(<.0001) * * * *$ & $(<.0001) * * *$ & $(<.0001)^{* * *}$ \\
\hline \multirow{2}{*}{$\begin{array}{l}\text { Liquid Assets Customer / Short Term Fund } \\
(-1)\end{array}$} & $\begin{array}{c}0.0630 \\
(<0001)^{* * * *}\end{array}$ & $\begin{array}{c}0.0536 \\
(<0001)^{* * *}\end{array}$ & $\begin{array}{c}0.0416 \\
(<0001) * * *\end{array}$ & $\begin{array}{c}0.1297 \\
(0.0155)^{* *}\end{array}$ \\
\hline & & 8.2635 & -6.0067 & 9.0315 \\
\hline \multirow[t]{2}{*}{ Herfindahl-Hirschman Index (-1) } & & $(0.0643)^{*}$ & $(0.2247)$ & $(0.0504)^{*}$ \\
\hline & & 0.1103 & 0.2686 & 0.2229 \\
\hline \multirow{3}{*}{$\begin{array}{l}\text { Market Share Conventional Banks (- 1) } \\
\text { Market Share Conventional Banks*Islamic } \\
\text { Bank Dummy (- 1) }\end{array}$} & & $(0.0233)^{* *}$ & $(<.0001)^{* * *}$ & $(0.0002) * * *$ \\
\hline & & & -0.1714 & -0.2721 \\
\hline & & & $\begin{array}{c}(\mathbf{0 . 1 8 8 2}) \\
8.0925\end{array}$ & $(0.0304)^{* *}$ \\
\hline Governance & & & $(<.0001)^{* * *}$ & \\
\hline \multirow{3}{*}{$\begin{array}{l}\text { LIBOR (- 1) } \\
\text { LIBOR* Islamic Bank Dummy } \\
(-1)\end{array}$} & & & & $\begin{array}{l}-0.1059 \\
(0.6493)\end{array}$ \\
\hline & & & & 1.3736 \\
\hline & & & & $\begin{array}{c}(\mathbf{0 . 0 7 4 8})^{*} \\
0.4321\end{array}$ \\
\hline \multirow[t]{2}{*}{ Real GDP Growth (-1) } & & & & $(0.0023)^{* * *}$ \\
\hline & & & & $\begin{array}{l}-0.1583 \\
(0.0077) * *\end{array}$ \\
\hline Inflation $(-1)$ & & & & $\begin{array}{l}(0.0077)^{* * * *} \\
-0.0812\end{array}$ \\
\hline \multirow[t]{2}{*}{ Official Exchange Rate (-1) } & & & & $(0.0427)^{* *}$ \\
\hline & -36.9669 & -49.6152 & -43.2630 & -63.6639 \\
\hline Constant & $(<.0001)^{* * *}$ & $(<.0001)^{* * *}$ & $(<.0001)^{* * *}$ & $(0.0001) * * *$ \\
\hline Observations & 1186 & 1186 & 1092 & 1069 \\
\hline R-Squared & 0.2796 & 0.2865 & 0.3622 & 0.3570 \\
\hline
\end{tabular}

Robust $P$ values in parentheses $*$ Significant at $10 \%$; * significant at $5 \%$; *** significant at $1 \%$.

Estimates of regression coefficients are reported in the following model: $\left(z_{i, j, t}\right)=\alpha+B_{i, j, t-1}+\gamma I_{j, t-1}+\sum \delta_{S} T_{S}+\sum \emptyset_{S} T_{S} I_{j, t-1}+\sum \varphi_{S} B_{i, j, t-1} T_{S}+\omega M_{j, t-1}+\varepsilon_{i, j, t}$. This table reports the regression results for the robust estimation with sample splits between MENA and Southeast Asia countries. The dependent variable is the z-score, a high index means a low bank insolvency risk exposure; thus the relationship between independent variables and bank insolvency risk exposure is reversed from the sign in this table. If Islamic banks were relatively weaker than conventional banks, the dummy variable would have a negative sign in the regression explaining z-scores. A negative sign for the interaction of independent variables and the Islamic bank dummy would indicate that a higher value reduces their soundness (reduces their zscores). 
Table 7. Robust Estimation (Large Banks - Middle East and North Africa) Least Trimmed Squares Parameter - Dependent Variable: Z-score

\begin{tabular}{|c|c|c|c|c|}
\hline \multirow{2}{*}{$\begin{array}{l}\text { Region/Country } \\
\text { Estimate no. }\end{array}$} & \multicolumn{4}{|c|}{ Middle East and North Africa } \\
\hline & (1) & (2) & (3) & (4) \\
\hline & 1.5897 & 0.0145 & 15.6499 & -7.7177 \\
\hline Islamic Bank Dummy & $(0.4188)$ & $(0.9942)$ & $(0.2353)$ & $(0.5692)$ \\
\hline & 1.6320 & 1.6370 & 2.3091 & 1.9113 \\
\hline Log Asset (-1) & $(<.0001)^{* * *}$ & $(<.0001) * * *$ & $(<.0001)^{* * * *}$ & $(<.0001)^{* * * *}$ \\
\hline & 0.0232 & 0.0222 & -0.0651 & -0.0034 \\
\hline Loans/ Assets (-1) & $(0.4624)$ & $(0.4851)$ & $(0.0942)^{*}$ & $(0.9334)$ \\
\hline & -0.0093 & -0.0100 & -0.0088 & -0.0093 \\
\hline Credit Risk (- 1) & $(0.0586)^{*}$ & $(0.0398)^{* *}$ & $(0.0858)^{*}$ & $(0.0572)^{*}$ \\
\hline Credit Risk * Islamic Bank Dummy & 0.0134 & -0.0406 & 0.0898 & 0.0878 \\
\hline$(-1)$ & $(\mathbf{0 . 7 4 3 3})$ & $(\mathbf{0 . 3 3 4 2})$ & $(0.0565) *$ & $(0.0586) *$ \\
\hline & -0.0977 & -0.0098 & -0.0087 & -1.4669 \\
\hline Income Diversity $(-1)$ & $(0.9266)$ & $(0.8952)$ & $(0.9106)$ & $(0.1837)$ \\
\hline $\begin{array}{l}\text { Income Diversity * Islamic Bank Dummy } \\
(-1)\end{array}$ & $\begin{array}{c}3.1383 \\
(\mathbf{0 . 2 7 4 8})\end{array}$ & $\begin{array}{c}4.9381 \\
(0.0711)^{*}\end{array}$ & $\begin{array}{c}4.0585 \\
(0.1543)\end{array}$ & $\begin{array}{c}6.2658 \\
(0.0820) *\end{array}$ \\
\hline & -0.1301 & -0.1131 & -0.1237 & -0.1800 \\
\hline Cost / Income Ratio (-1) & $(<.0001) * * *$ & $(<.0001)^{* * * *}$ & $(<.0001)^{* * *}$ & $(<.0001)^{* * * *}$ \\
\hline Liquid Assets Customer / Short Term Fund & 0.0230 & 0.0328 & -0.0336 & 0.0260 \\
\hline$(-1)$ & $(0.0514)^{*}$ & $(<.0001)^{* * *}$ & $(0.2494)$ & $(0.4500)$ \\
\hline & & -4.2430 & -7.2067 & -1.6226 \\
\hline Herfindahl-Hirschman Index (-1) & & $(0.4581)$ & $(0.2686)$ & $(0.7937)$ \\
\hline & & 0.0461 & 0.3146 & 0.1316 \\
\hline Market Share Conventional Banks (- 1) & & $(0.4782)$ & $(0.0003) * * *$ & $(0.1193)$ \\
\hline Market Share Conventional Banks*Islamic & & & -0.1882 & -0.0120 \\
\hline Bank Dummy $(-1)$ & & & $\begin{array}{c}(\mathbf{0 . 2 1 5 3}) \\
8.1699\end{array}$ & $(0.9348)$ \\
\hline Governance & & & $(<.0001)^{* * * *}$ & \\
\hline LIBOR (-1) & & & & $\begin{array}{c}-0.2124 \\
(0.4490)\end{array}$ \\
\hline LIBOR* Islamic Dummy & & & & 1.7556 \\
\hline$(-1)$ & & & & $(0.0190)^{* * *}$ \\
\hline Real GDP Growth $(-1)$ & & & & $(0.0025)^{* * *}$ \\
\hline & & & & -0.0392 \\
\hline Inflation $(-1)$ & & & & $(0.5130)$ \\
\hline Official Exchange Rate (-1) & & & & $\begin{array}{l}-0.0317 \\
(0.4242)\end{array}$ \\
\hline & -3.1913 & -6.3434 & -34.0406 & -16.0096 \\
\hline Constant & (0.6795) & $(0.5131)$ & $(0.0034) * * *$ & $(0.1889)$ \\
\hline Observations & 703 & 703 & 643 & 588 \\
\hline R-Squared & 0.2369 & 0.2629 & 0.2851 & 0.3484 \\
\hline
\end{tabular}


Table 8. Robust Estimation (Large Banks - Southeast Asia and Pakistan) Least Trimmed Squares Parameter - Dependent Variable: Z-score

\begin{tabular}{|c|c|c|c|c|}
\hline \multirow{2}{*}{$\begin{array}{l}\text { Region/Country } \\
\text { Estimate no. }\end{array}$} & \multicolumn{4}{|c|}{ Southeast Asia and Pakistan } \\
\hline & (1) & (2) & (3) & (4) \\
\hline \multirow{3}{*}{ Islamic Bank Dummy } & 29.8441 & 30.1486 & 3.0197 & 110.7513 \\
\hline & $(<.0001)^{* * *}$ & $(<.0001) * * *$ & $(\mathbf{0 . 8 9 3 3 )}$ & $(0.0003)^{* * *}$ \\
\hline & 5.9120 & 5.6824 & 2.7381 & 4.5289 \\
\hline \multirow{2}{*}{ Log Asset (-1) } & $(0.0007)^{* * *}$ & $(<.0001)^{* * *}$ & $(<.0001)^{* * *}$ & $(<.0001) * * *$ \\
\hline & 0.3316 & 0.3406 & 0.2426 & 0.2677 \\
\hline \multirow[t]{2}{*}{ Loans/ Assets (-1) } & $(<.0001)^{* * *}$ & $(<.0001) * * *$ & $(<.0001)^{* * * *}$ & $(<.0001)^{* * *}$ \\
\hline & -0.0003 & -0.0002 & 0.0004 & -0.0003 \\
\hline Credit Risk (- 1) & $(0.8684)$ & $(0.9057)$ & $(0.7966)$ & $(0.8159)$ \\
\hline Credit Risk * Islamic Bank Dummy & -0.3969 & -0.3874 & -0.4224 & -0.5910 \\
\hline \multirow{2}{*}{$(-1)$} & $(0.0523) *$ & $(0.0584) *$ & $(0.0265) * *$ & $(0.0053) * * *$ \\
\hline & -0.4271 & -0.5849 & -2.1337 & -1.1236 \\
\hline Income Diversity $(-1)$ & $(0.7330)$ & $(0.6412)$ & $(0.1304)^{* * * *}$ & $(0.3570)$ \\
\hline \multirow{2}{*}{$\begin{array}{l}\text { Income Diversity * Islamic Bank Dummy } \\
(-1)\end{array}$} & -25.4954 & -25.0508 & -15.4124 & -26.9590 \\
\hline & $(0.0083) * * *$ & $(0.0117)^{* *}$ & (0.1197) & $(0.0138)^{* *}$ \\
\hline Cost / Income Ratio (-1) & $(0.0004)^{* * * *}$ & $(0.0002) * * *$ & $(<.0001)^{*} * * *$ & $(0.0345$ * \\
\hline \multirow{3}{*}{$\begin{array}{l}\text { Liquid Assets Customer / Short Term Fund } \\
\text { (-1) }\end{array}$} & 0.2966 & 0.2999 & 0.1705 & 0.2569 \\
\hline & $(<.0001)^{* * *}$ & $(<.0001)^{* * *}$ & $(0.0001)^{* * *}$ & $(<.0001)^{* * *}$ \\
\hline & & 24.8823 & 16.9407 & 13.8123 \\
\hline \multirow[t]{2}{*}{ Herfindahl-Hirschman Index (-1) } & & $(0.0022)^{* * *}$ & $(0.0964)^{*}$ & $(0.0943)^{*}$ \\
\hline & & 0.1630 & -0.1666 & 0.2001 \\
\hline Market Share Conventional Banks (- 1) & & $(0.0953) *$ & $(0.3724)$ & $(0.0906)^{*}$ \\
\hline Market Share Conventional Banks*Islamic & & & 0.1926 & -1.1498 \\
\hline Bank Dummy $(-1)$ & & & $\begin{array}{l}(\mathbf{0 . 4 2 7 3}) \\
8.3201\end{array}$ & $(0.0135)^{* *}$ \\
\hline \multirow{2}{*}{ Governance } & & & $(<.0001)^{* * * *}$ & \\
\hline & & & & $\begin{array}{l}-0.1222 \\
(0.7446)\end{array}$ \\
\hline \multirow[t]{2}{*}{$\begin{array}{l}\text { LIBOR* Islamic Bank Dummy } \\
(-1)\end{array}$} & & & & $\begin{array}{l}6.5341 \\
(0.1654)\end{array}$ \\
\hline & & & & 0.4950 \\
\hline \multirow{2}{*}{ Real GDP Growth (-1) } & & & & $(0.1919)$ \\
\hline & & & & -0.6775 \\
\hline \multirow[t]{2}{*}{ Inflation (-1) } & & & & $(0.0002) * * *$ \\
\hline & & & & $\begin{array}{c}-0.3033 \\
(0.0027) * * *\end{array}$ \\
\hline Official Exchange Rate (-1) & -95.3330 & -111.162 & -20.5422 & $\begin{array}{l}(0.0027) \\
-89.1692\end{array}$ \\
\hline Constant & $(<.0001)^{* * *}$ & $(<.0001)^{* * *}$ & $(0.3652)$ & $(<.0001)^{* * *}$ \\
\hline Observations & 483 & 483 & 449 & 481 \\
\hline R-Squared & 0.4792 & 0.4875 & 0.5415 & 0.5193 \\
\hline
\end{tabular}


Table 9. Robust Estimation (Small Banks - All Countries) Least Trimmed Squares Parameter - Dependent Variable : Z-score

\begin{tabular}{|c|c|c|c|c|}
\hline \multirow{2}{*}{$\begin{array}{l}\text { Region/Country } \\
\text { Estimate no. }\end{array}$} & \multicolumn{4}{|c|}{ All Countries } \\
\hline & (1) & (2) & (3) & (4) \\
\hline Islamic Bank Dummy & $\begin{array}{c}-8.4574 \\
(0.00941)^{* * * *}\end{array}$ & $\begin{array}{l}-5.8727 \\
(0.0669) *\end{array}$ & $\begin{array}{l}-1.8487 \\
(0.7953)\end{array}$ & $\begin{array}{l}-0.4078 \\
(0.9571)\end{array}$ \\
\hline 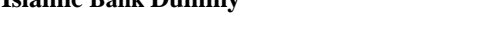 & -1.8820 & $\begin{array}{l}-1.9998 \\
-1.998\end{array}$ & -1.8038 & -1.5661 \\
\hline Log Asset (-1) & $(0.0001) * * *$ & $(<.0001)^{* * *}$ & $(0.0007)^{* * *}$ & $(0.0009)^{* * *}$ \\
\hline & 0.0037 & -0.0445 & -0.0423 & -0.0490 \\
\hline Loans/ Assets (-1) & $(0.8573)$ & $(0.0342)^{* *}$ & $(0.0638)^{*}$ & $(0.0228)^{* *}$ \\
\hline Credit Risk $(-1)$ & $\begin{array}{c}0.0002 \\
(0.8786)\end{array}$ & $\begin{array}{c}-0.0036 \\
(0.0076)^{* * *}\end{array}$ & $\begin{array}{c}-0.0097 \\
(0.0015)^{* * *}\end{array}$ & $\begin{array}{c}-0.0145 \\
(<.0001)^{* * *}\end{array}$ \\
\hline $\begin{array}{l}\text { Credit Risk * Islamic Bank Dummy } \\
(-1)\end{array}$ & $\begin{array}{l}-0.0290 \\
(0.0712) *\end{array}$ & $\begin{array}{l}-0.0214 \\
(0.1636)\end{array}$ & $\begin{array}{c}0.0103 \\
(0.0019) * * *\end{array}$ & $\begin{array}{l}-0.0051 \\
(0.8017)\end{array}$ \\
\hline Income Diversity (-1) & $\begin{array}{c}0.0172 \\
(0.9266)\end{array}$ & $\begin{array}{c}0.0071 \\
(0.9683)\end{array}$ & $\begin{array}{l}-0.0258 \\
(0.8876)\end{array}$ & $\begin{array}{l}-0.0688 \\
(0.7040)\end{array}$ \\
\hline $\begin{array}{l}\text { Income Diversity * Islamic Bank Dummy } \\
(-1)\end{array}$ & $\begin{array}{l}15.5730 \\
(0.0009) * * *\end{array}$ & $\frac{12.8206}{(0.0046) * * *}$ & $\begin{array}{l}11.0466 \\
(0.0213) * *\end{array}$ & $\begin{array}{c}14.2898 \\
(0.0091) * * *\end{array}$ \\
\hline Cost / Income Ratio (-1) & $\begin{array}{c}-0.0960 \\
(<.0001)^{* * *}\end{array}$ & $\begin{array}{c}-0.1238 \\
(<.0001)^{* * *}\end{array}$ & $\begin{array}{c}-0.0885 \\
(<.0001)^{* * * *}\end{array}$ & $\begin{array}{c}-0.1063 \\
(<.0001)^{* * * *}\end{array}$ \\
\hline $\begin{array}{l}\text { Liquid Assets Customer / Short Term Fund } \\
(-1)\end{array}$ & $\begin{array}{c}0.0553 \\
(<.0001)^{* * *}\end{array}$ & $\begin{array}{l}0.0551 \\
(<.0001)^{* * *} \\
-25.6624\end{array}$ & $\begin{array}{l}0.1255 \\
(<.0001)^{* * *} \\
-31.5252\end{array}$ & $\begin{array}{l}0.0583 \\
(<.0001)^{* * *} \\
-28.4439\end{array}$ \\
\hline Herfindahl-Hirschman Index (-1) & & $\begin{array}{l}-25.6624 \\
(<.0001)^{* * *} \\
0.0744\end{array}$ & $\begin{array}{l}-31.5252 \\
(<.0001)^{* * *} \\
0.0959\end{array}$ & $\begin{array}{l}-28.4439 \\
(<.0001)^{* * *} \\
0.0883\end{array}$ \\
\hline Market Share Conventional Banks (- 1) & & $(0.0345)^{* *}$ & $(0.0268)^{* *}$ & $(0.0278)^{* *}$ \\
\hline $\begin{array}{l}\text { Market Share Conventional Banks*Islamic } \\
\text { Bank Dummy }(-1)\end{array}$ & & & $\begin{array}{c}-0.0566 \\
(0.4774)\end{array}$ & $\begin{array}{l}-0.0186 \\
(0.8148)\end{array}$ \\
\hline Governance & & & $\begin{array}{c}0.6638 \\
(0.4742)\end{array}$ & \\
\hline LIBOR (- 1) & & & & $\begin{array}{c}0.4390 \\
(0.0454)^{* *}\end{array}$ \\
\hline $\begin{array}{l}\text { LIBOR* Islamic Bank Dummy } \\
(-1)\end{array}$ & & & & $\begin{array}{l}-1.4348 \\
(0.0718)^{*}\end{array}$ \\
\hline Real GDP Growth (-1) & & & & $\begin{array}{l}-0.4716 \\
(0.0202)^{* *} \\
-0.0043\end{array}$ \\
\hline Inflation (-1) & & & & $(0.9375)$ \\
\hline Official Exchange Rate (-1) & & & & $\begin{array}{c}-0.1266 \\
(0.0019) * * *\end{array}$ \\
\hline Constant & $\begin{array}{c}43.0742 \\
(<.0001)^{* * * *}\end{array}$ & $\begin{array}{c}45.0180 \\
(<.0001)^{* * *}\end{array}$ & $\begin{array}{c}41.8684 \\
(<.0001)^{* * * *}\end{array}$ & $\begin{array}{c}39.3616 \\
(<.0001)^{* * * *}\end{array}$ \\
\hline Observations & 873 & 873 & 764 & 855 \\
\hline R-Squared & 0.1659 & 0.2022 & 0.2192 & 0.2512 \\
\hline
\end{tabular}

Robust $P$ values in parentheses * Significant at 10\%; ** significant at $5 \%$; ** significant at $1 \%$.

Estimates of regression coefficients are reported in the following model: $\left(z_{i, j, t}\right)=\alpha+\varphi B_{i, j, t-1}+\gamma I_{j, t-1}+\sum \delta_{S} T_{S}+\sum \emptyset_{S} T_{S} I_{j, t-1}+\sum \varphi_{S} B_{i, j, t-1} T_{S}+\omega M_{j, t-1}+\varepsilon_{i, j, t}$. This table reports the regression results for the robust estimation with sample splits between MENA and Southeast Asia countries. The dependent variable is the z-score, a high index means a low bank insolvency risk exposure; thus the relationship between independent variables and bank insolvency risk exposure is reversed from the sign in this table. If Islamic banks were relatively weaker than conventional banks, the dummy variable would have a negative sign in the regression explaining z-scores. A negative sign for the interaction of independent variables and the Islamic bank dummy would indicate that a higher value reduces their soundness (reduces their $z$ scores). 
Table 10. Robust Estimation (Small Banks - Middle East and North Africa) Least Trimmed Squares Parameter - Dependent Variable : Z-score

\begin{tabular}{|c|c|c|c|c|}
\hline \multirow{2}{*}{$\begin{array}{l}\text { Region/Country } \\
\text { Estimate no. }\end{array}$} & \multicolumn{4}{|c|}{ Middle East and North Africa } \\
\hline & (1) & (2) & (3) & (4) \\
\hline & -3.3683 & -3.1425 & -22.2046 & -24.3112 \\
\hline Islamic Bank Dummy & $(0.2347)$ & $(0.2850)$ & $(0.0014)^{* * *}$ & $(0.0004)^{* * *}$ \\
\hline & -1.4981 & -1.6265 & -1.4968 & -1.8234 \\
\hline Log Asset (-1) & $(0.0207)^{* *}$ & $(0.0219)^{* *}$ & $(0.0566)^{*}$ & $(0.0099)^{* * *}$ \\
\hline & 0.0300 & 0.0232 & 0.0906 & 0.0376 \\
\hline Loans/ Assets ( -1$)$ & $(0.2854)$ & $(0.4624)$ & $(0.0062)^{* * *}$ & $(0.2121)$ \\
\hline & -0.0121 & -0.0128 & -0.0044 & -0.0141 \\
\hline Credit Risk (- 1) & $(0.0046)^{* * *}$ & $(0.0032)^{* * *}$ & $(0.1819)$ & $(0.0014)^{* * *}$ \\
\hline Credit Risk * Islamic Bank Dummy & $-\mathbf{0 . 0 3 3 0}$ & -0.0328 & -0.0238 & -0.0213 \\
\hline$(-1)$ & $(0.0184) * *$ & $(0.0197)^{* *}$ & $(0.1016)$ & $(\mathbf{0 . 2 1 8 3 )}$ \\
\hline & 0.1755 & -0.3329 & -0.3230 & -0.2060 \\
\hline Income Diversity $(-1)$ & $(0.7648)$ & $(0.1900)$ & $(0.2271)$ & $(0.4241)$ \\
\hline Income Diversity * Islamic Bank Dummy & 17.5259 & 17.6820 & 7.3217 & 15.9814 \\
\hline$(-1)$ & $\begin{array}{c}(<.0001)^{* * * *} \\
-0.1130\end{array}$ & $\begin{array}{c}(<.0001)^{* * * *} \\
-0.1407\end{array}$ & $\begin{array}{c}(\mathbf{0 . 0 8 6 0})^{*} \\
-0.1158\end{array}$ & $\begin{array}{c}(\mathbf{0 . 0 0 1 2}) * * * \\
-0.0978\end{array}$ \\
\hline Cost / Income Ratio (-1) & $(<.0001)^{* * *}$ & $(<.0001)^{* * *}$ & $(<.0001)^{* * *}$ & $(<.0001)^{* * *}$ \\
\hline Liquid Assets Customer / Short Term Fund & 0.0078 & 0.0067 & 0.0870 & 0.0901 \\
\hline$(-1)$ & $(0.6112)$ & $(0.6744)$ & $(<.0001)^{* * *}$ & $(<.0001)^{* * *}$ \\
\hline & & -5.4040 & -0.4194 & -2.6636 \\
\hline Herfindahl-Hirschman Index (-1) & & $(0.4542)$ & $(0.9601)$ & $(0.7184)$ \\
\hline & & 0.0224 & -0.0521 & -0.0354 \\
\hline Market Share Conventional Banks (- 1) & & $(0.5825)$ & $(0.3239)$ & $(0.4310)$ \\
\hline Market Share Conventional Banks*Islamic & & & 0.3390 & 0.2560 \\
\hline Bank Dummy (- 1) & & & $\begin{array}{c}(<.0001) * * * \\
-1.3755\end{array}$ & $(0.0009) * * *$ \\
\hline Governance & & & $(0.3999)$ & \\
\hline $\operatorname{LIBOR}(-1)$ & & & & $\begin{array}{c}0.1705 \\
(0.5969)\end{array}$ \\
\hline LIBOR* Islamic Bank Dummy & & & & 0.7714 \\
\hline 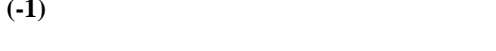 & & & & $\begin{array}{l}(\mathbf{0 . 3 1 8 6 )} \\
-0.7859\end{array}$ \\
\hline Real GDP Growth (-1) & & & & $(0.0004)^{* * *}$ \\
\hline & & & & -0.0726 \\
\hline Inflation $(-1)$ & & & & $(0.1859)$ \\
\hline Official Exchange Rate (-1) & & & & $\begin{array}{l}-0.0731 \\
(0.1042)\end{array}$ \\
\hline & 36.7312 & 38.1473 & 35.4897 & 45.5562 \\
\hline Constant & $(<.0001)^{* * * *}$ & $(<.0001)^{* * *}$ & $(0.0013)^{* * *}$ & $(<.0001)^{* * *}$ \\
\hline Observations & 321 & 321 & 278 & 303 \\
\hline R-Squared & 0.2391 & 0.2462 & 0.2622 & 0.3412 \\
\hline
\end{tabular}


Table 11. Robust Estimation (Small Banks - Southeast Asia and Pakistan) Least Trimmed Squares Parameter - Dependent Variable : Z-score

\begin{tabular}{|c|c|c|c|c|}
\hline \multirow{2}{*}{$\begin{array}{l}\text { Region/Country } \\
\text { Estimate no. }\end{array}$} & \multicolumn{4}{|c|}{ Southeast Asia and Pakistan } \\
\hline & (1) & (2) & (3) & (4) \\
\hline \multirow{3}{*}{ Islamic Bank Dummy } & -16.4357 & 40.1367 & 109.6306 & 441.5086 \\
\hline & $(0.0979) *$ & $(<.0001)^{* * * *}$ & $(0.7321)$ & $(0.3629)$ \\
\hline & -0.5663 & -0.9993 & -1.1402 & -1.3024 \\
\hline \multirow[t]{2}{*}{ Log Asset (-1) } & $(0.3757)$ & $(0.1214)$ & $(0.1328)$ & $(0.0411)^{* *}$ \\
\hline & -0.0831 & -0.1073 & -0.1093 & -0.1496 \\
\hline \multirow[t]{2}{*}{ Loans/ Assets (-1) } & $(0.0047)^{* * *}$ & $(0.0003)^{* * *}$ & $(0.0012)^{* * *}$ & $(<.0001)^{* * *}$ \\
\hline & 0.0017 & 0.0010 & -0.0052 & -0.0024 \\
\hline Credit Risk (- 1) & $(0.1153)$ & $(0.3796)$ & $(0.4206)$ & $(0.1236)$ \\
\hline \multirow{2}{*}{$\begin{array}{l}\text { Credit Risk * Islamic Bank Dummy } \\
(-1)\end{array}$} & 0.6371 & 0.2059 & 0.2197 & -0.0878 \\
\hline & $\begin{array}{l}(\mathbf{0 . 2 3 2 0}) \\
-5.4887\end{array}$ & $\begin{array}{l}(\mathbf{0 . 6 1 9 4}) \\
-5.9936\end{array}$ & $\begin{array}{l}(\mathbf{0 . 6 1 3 7}) \\
-5.7791\end{array}$ & $\begin{array}{l}(\mathbf{0 . 8 3 7 2}) \\
-4.8260\end{array}$ \\
\hline Income Diversity (-1) & $(<.0001)^{* * *}$ & $(<.0001)^{* * *}$ & $(0.0004)^{* * *}$ & $(0.0005)^{* * *}$ \\
\hline \multirow[t]{2}{*}{$\begin{array}{l}\text { Income Diversity * Islamic Bank Dummy } \\
(-1)\end{array}$} & $\begin{array}{c}7.3488 \\
(0.6300)\end{array}$ & $\begin{array}{c}-67.9937 \\
(<.0001)^{* * * *}\end{array}$ & $\begin{array}{c}-67.4792 \\
(<.0001)^{* * * *}\end{array}$ & $\begin{array}{c}-49.5890 \\
(0.0001) * * *\end{array}$ \\
\hline & -0.0960 & -0.1020 & -0.0716 & -0.1529 \\
\hline Cost / Income Ratio (-1) & $(<.0001)^{* * *}$ & $(<.0001)^{* * *}$ & $(<.0001)^{* * *}$ & $(<.0001)^{* * *}$ \\
\hline \multirow[t]{2}{*}{$\begin{array}{l}\text { Liquid Assets Customer / Short Term Fund } \\
(-1)\end{array}$} & $\begin{array}{c}0.1423 \\
(<.0001)^{* * *}\end{array}$ & $\begin{array}{c}0.1478 \\
(<.0001)^{* * * *}\end{array}$ & $\begin{array}{c}0.1542 \\
(<.0001)^{* * *}\end{array}$ & $\begin{array}{c}0.0394 \\
(<.0001)^{* * * *}\end{array}$ \\
\hline & & -28.1680 & -58.2572 & -27.2961 \\
\hline \multirow[t]{2}{*}{ Herfindahl-Hirschman Index (-1) } & & $(0.0003)^{* * *}$ & $(<.0001) * * *$ & $(0.0010)^{* * *}$ \\
\hline & & -0.8020 & 0.1282 & -0.8559 \\
\hline Market Share Conventional Banks (- 1) & & $(<.0001)^{* * * *}$ & $(0.4874)$ & $(<.0001)^{* * *}$ \\
\hline \multirow{2}{*}{$\begin{array}{l}\text { Market Share Conventional Banks*Islamic } \\
\text { Bank Dummy (- 1) }\end{array}$} & & & -0.7014 & -3.9982 \\
\hline & & & $\begin{array}{c}(\mathbf{0 . 8 3 0 7}) \\
4.4665\end{array}$ & $(0.4114)$ \\
\hline \multirow[t]{2}{*}{ Governance } & & & $(0.0017)^{* * *}$ & \\
\hline & & & & $\begin{array}{c}0.2812 \\
(0.3286)\end{array}$ \\
\hline \multirow{2}{*}{$\begin{array}{l}\text { LIBOR* Islamic Bank Dummy } \\
(-1)\end{array}$} & & & & -5.4636 \\
\hline & & & & $\begin{array}{c}(\mathbf{0 . 0 4 0 0})^{* *} \\
0.1066\end{array}$ \\
\hline \multirow[t]{2}{*}{ Real GDP Growth (-1) } & & & & $(0.8090)$ \\
\hline & & & & 0.3909 \\
\hline \multirow[t]{2}{*}{ Inflation (-1) } & & & & $(0.0185)^{* *}$ \\
\hline & & & & -0.0842 \\
\hline \multirow[t]{2}{*}{ Official Exchange Rate (-1) } & & & & $(0.2678)$ \\
\hline & 37.3279 & 127.3751 & 45.1546 & 135.7748 \\
\hline Constant & $(<.0001) * * *$ & $(<.0001) * * *$ & $(0.0444)^{* *}$ & $(<.0001)^{* * *}$ \\
\hline Observations & 552 & 552 & 486 & 552 \\
\hline R-Squared & 0.2567 & 0.2897 & 0.3367 & 0.3446 \\
\hline
\end{tabular}

Robust $P$ values in parentheses $*$ Significant at $10 \%$; * significant at $5 \%$; *** significant at $1 \%$. 
Table 12. Quantile Regression Results (Full sample) Dependent Variable : Z-score Logged

\begin{tabular}{|c|c|c|c|c|c|c|}
\hline \multirow[b]{2}{*}{ Quantile } & \multicolumn{3}{|c|}{$\begin{array}{c}\text { Middle East and } \\
\text { North Africa }\end{array}$} & \multicolumn{3}{|c|}{$\begin{array}{c}\text { Southeast Asia and } \\
\text { Pakistan }\end{array}$} \\
\hline & $\begin{array}{c}0.25 \\
(1)\end{array}$ & $\begin{array}{l}0.5 \\
(2)\end{array}$ & $\begin{array}{c}0.75 \\
(3)\end{array}$ & $\begin{array}{c}0.25 \\
(1)\end{array}$ & $\begin{array}{l}0.5 \\
(2)\end{array}$ & $\begin{array}{c}\mathbf{0 . 7 5} \\
(3)\end{array}$ \\
\hline Islamic Bank Dummy & $\begin{array}{l}-1.3286 \\
(0.1069)\end{array}$ & $\begin{array}{r}-0.8672 \\
(0.1063)\end{array}$ & $\begin{array}{l}-0.1778 \\
(0.7243)\end{array}$ & $\begin{array}{c}2.0862 \\
(0.3865)\end{array}$ & $\begin{array}{c}4.1768 \\
(0.0619) *\end{array}$ & $\begin{array}{l}1.5198 \\
(0.5267)\end{array}$ \\
\hline Log Asset & 0.0904 & 0.0201 & 0.0142 & -0.0035 & 0.0059 & 0.0160 \\
\hline$(-1)$ & $(0.0028) * * *$ & $(0.3415)$ & $(0.5675)$ & $(0.9128)$ & $(0.7598)$ & $(0.4125)$ \\
\hline Loans/ Assets & 0.0010 & 0.0013 & 0.0008 & -0.0030 & 0.0001 & -0.0022 \\
\hline$(-1)$ & $(0.7403)$ & $(0.5902)$ & $(0.7842)$ & $(0.2428)$ & $(0.9694)$ & $(0.4003)$ \\
\hline Credit Risk & -0.0022 & -0.0018 & -0.0006 & -0.0015 & 0.0001 & 0.0000 \\
\hline$(-1)$ & $(0.1013)$ & $(0.0165)^{* *}$ & $(0.5183)$ & $(0.9617)$ & $(0.6824)$ & $(0.9625)$ \\
\hline Credit Risk * Islamic Bank & 0.0023 & 0.0018 & 0.0005 & -0.0145 & -0.0137 & -0.0175 \\
\hline Dummy (-1) & $(0.1379)$ & $(0.0426)^{* *}$ & $(0.6250)$ & $(0.3264)$ & $(0.2811)$ & $(0.2072)$ \\
\hline $\begin{array}{l}\text { Income Diversity } \\
(-1)\end{array}$ & $\begin{array}{l}-0.0982 \\
(0.1816)\end{array}$ & $\begin{array}{l}-0.0571 \\
(0.4167)\end{array}$ & $\begin{array}{l}-0.0159 \\
(0.8705)\end{array}$ & $\begin{array}{l}-0.0444 \\
(0.7974)\end{array}$ & $\begin{array}{r}0.0219 \\
(0.9016)\end{array}$ & $\begin{array}{l}-0.0114 \\
(0.9434)\end{array}$ \\
\hline $\begin{array}{l}\text { Income Diversity * Islamic } \\
\text { Bank Dummy (-1) }\end{array}$ & $\begin{array}{c}\mathbf{0 . 7 5 4 9} \\
(\mathbf{0 . 0 3 7 3})^{* *}\end{array}$ & $\begin{array}{c}0.3425 \\
(\mathbf{0 . 1 6 9 5})\end{array}$ & $\begin{array}{l}-\mathbf{0 . 0 1 9 6} \\
(\mathbf{0 . 9 3 3 7})\end{array}$ & $\begin{array}{l}-2.5448 \\
(0.0022)^{* * *}\end{array}$ & $\begin{array}{l}-1.4633 \\
(0.0804) *\end{array}$ & $\begin{array}{c}-2.1158 \\
(0.0388)^{* *}\end{array}$ \\
\hline Cost / Income Ratio & -0.0129 & -0.0088 & -0.0043 & -0.0029 & -0.0090 & -0.0050 \\
\hline$(-1)$ & $(0.0004) * * *$ & $(<.0001)^{* * *}$ & $(0.0596)^{*}$ & $(<.0001) * * *$ & $(<.0001) * * *$ & $(<.0001) * * *$ \\
\hline Liquid Assets Customer/ & 0.0030 & 0.0021 & 0.0016 & 0.0023 & 0.0043 & 0.0043 \\
\hline Short Term Fund (-1) & $(<.0001)^{* * *}$ & $(0.0599)^{*}$ & $(0.3214)$ & $(0.6079)$ & $(0.0001) * * *$ & $(0.0369)^{* *}$ \\
\hline Herfindahl-Hirschman Index & -1.1520 & 0.0154 & -0.6799 & 0.0520 & -0.1833 & 0.2984 \\
\hline & $(0.0694)^{*}$ & $(0.9734)$ & $(0.1048)$ & $(0.9324)$ & $(0.7403)$ & $(0.5573)$ \\
\hline Market Share Conventional & -0.0125 & -0.0031 & 0.0039 & 0.0122 & 0.0207 & 0.0045 \\
\hline Banks (-1) & $(0.0102)^{* *}$ & $(0.3617)$ & $(0.2380)$ & $(0.1026)$ & $(0.0214)^{* *}$ & $(0.6575)$ \\
\hline \multirow{3}{*}{$\begin{array}{l}\text { Market Share Conventional } \\
\text { Banks*Islamic Dummy (- 1) }\end{array}$} & 0.0105 & 0.0087 & 0.0042 & 0.0055 & -0.0267 & 0.0007 \\
\hline & $(0.2224)$ & $(0.1067)$ & $(\mathbf{0 . 4 1 7 2})$ & $(0.8169)$ & $(0.2652)$ & $(\mathbf{0 . 9 8 0 0 )}$ \\
\hline & -0.0138 & 0.0169 & 0.0269 & 0.0396 & 0.0238 & 0.0193 \\
\hline LIBOR (-1) & $(0.5215)$ & $(0.3395)$ & $(0.3018)$ & $(0.1597)$ & $(0.2169)$ & $(0.2956)$ \\
\hline LIBOR* Islamic Dummy & 0.0661 & 0.0368 & -0.0123 & -0.2573 & -0.1129 & -0.0625 \\
\hline \multirow[t]{2}{*}{$(-1)$} & $(\mathbf{0 . 1 7 3 7})$ & $(0.4076)$ & $(0.7854)$ & $(0.2874)$ & $(0.3559)$ & $(0.7244)$ \\
\hline & -0.0001 & 0.0173 & 0.0157 & 0.0221 & 0.0565 & 0.0191 \\
\hline \multirow[t]{2}{*}{ Real GDP Growth (-1) } & $(0.9910)$ & $(0.0743)^{*}$ & $(0.2014)$ & $(0.1365)$ & $(0.0815)^{*}$ & $(0.3590)$ \\
\hline & -0.0089 & -0.0081 & -0.0066 & -0.0163 & -0.0318 & -0.0346 \\
\hline \multirow[t]{2}{*}{ Inflation (-1) } & $(0.0633)^{*}$ & $(0.0589)^{*}$ & $(0.2519)$ & $(0.2125)$ & $(0.0033) * * *$ & $(0.0005) * * *$ \\
\hline & 0.0003 & -0.0068 & -0.0088 & -0.0188 & -0.0262 & -0.0156 \\
\hline \multirow[t]{2}{*}{ Official Exchange Rate (-1) } & $(0.9439)$ & $(0.0386)^{* *}$ & $(0.0654)^{*}$ & $(0.0030)^{* * * *}$ & $(<.0001)^{* * *}$ & $(0.0010) * * *$ \\
\hline & 2.8804 & 3.0214 & 2.8461 & 1.6314 & 0.8691 & 2.9497 \\
\hline Constant & $(<.0001)^{* * *}$ & $(<.0001)^{* * *}$ & $(<.0001)^{* * *}$ & $(0.0873)^{*}$ & $(0.3747)$ & $(0.0091)^{* * *}$ \\
\hline Observations & 879 & 879 & 879 & 1014 & 1014 & 1014 \\
\hline
\end{tabular}

$P$ values in parentheses $*$ Significant at 10\%; ** significant at 5\%; *** significant at $1 \%$.

Estimates of quantile regression coefficients are reported in the following model:

$\log \left(z_{i, j, t}\right)=\alpha+\varphi B_{i, j, t-1}+\gamma I_{j, t-1}+\sum \delta_{S} T_{S}+\sum \emptyset_{S} T_{S} I_{j, t-1}+\sum \varphi_{S} B_{i, j, t-1} T_{S}+\omega M_{j, t-1}+\varepsilon_{i, j, t}$.

This table reports the regression results for the quantile estimation with sample splits between

MENA and Southeast Asia countries. The dependent variable is the z-score logged, a high index means a low bank insolvency risk exposure; thus the relationship between independent variables and bank insolvency risk exposure is reversed from the sign in this table. If Islamic banks were relatively weaker than conventional banks, the dummy variable would have a negative sign in the regression explaining z-scores. A negative sign for the interaction of independent variables and the Islamic bank dummy would indicate that a higher value reduces their soundness (reduces their z-scores). 
We find that a higher share of loans in the asset structure contributes to increasing bank insolvency for large banks in MENA countries in specification (3) at the 10 percent level. The slope coefficient is consistently negative for small banks in Southeast Asian countries in all specifications and significant at the 1 percent level. Nevertheless, a higher share of loans contributes to decreasing bank insolvency for large banks in Southeast Asian countries in all specification at the 1 percent level. The slope coefficient is consistently positive for small banks in MENA countries in specification (3) at the 1 percent level.

Our assumption that credit risk contributes to the instability of the Islamic banking sector is confirmed for large banks in Southeast Asian countries. The results are negative in specifications (1), (2), (3) and (4) at the 1, 5 and 10 percent levels. The results indicate that credit risk for small Islamic banks in MENA countries is on average negatively related to bank stability in specifications (1) and (2) at the 5 percent level. Our results indicate that credit risk for large Islamic banks in MENA countries is positively related to bank stability in specifications (3) and (4) at the 10 percent level. Islamic banks have the greatest exposure to credit risk. This risk would increase if lending were to expand rapidly before quality deteriorates. We find a negative relationship between $\mathrm{z}$-score seems to be inversely related to credit risk, probably due to the absence of enhanced screening and monitoring of borrowers. This might suggest that Islamic banks in a given period reflect non-performing loans rather than good risk management practice.

Our results are consistent with Čihák and Hesse (2010). These authors show that the smaller banks tend to focus on lower risk investments, whereas more PLS financing is conducted by large Islamic banks. The suggested explanation for these characteristics is that Islamic banks tend to have problems monitoring credit risks when the business becomes bigger and therefore more complex. The limits of this credit risk management at a larger scale mean problems with moral hazard. Thus, potential distress continues to be a poor portfolio risk management, despite the prudential regulation or a lack of attention to changing external circumstances that can adversely affect the credit standing of a bank's counterparties. Ariss (2010) finds that between 2000 and 2006, Islamic banks allocated a significantly greater share of their assets to financing compared to conventional banks, implying a greater exposure to credit risk. ${ }^{17}$ In turn, Islamic banks balance the higher portfolio risk with significantly lower financial risk through higher capitalization levels.

Our econometric results show that the evidence regarding the impact of revenue diversification on insolvency risk in each region and group is mixed. There is a significant difference in terms of income diversity between Islamic and conventional banks (large or small) in each region. With a strongly positive coefficient, greater income diversity tends to increase z-scores in large and

\footnotetext{
${ }^{17}$ The credit risk management in Islamic banks is more complicated not only due to the nature of the contract and additional externalities. Islamic banks cannot charge a penalty due to default in payment whereas conventional banks can charge penalties for overdue interest.
} 
small Islamic banks in MENA countries at the 1,5 and 10 percent levels, suggesting that a move from lending-based operations to other sources of income might improve stability in those banks. Greater income diversity tends to decrease z-scores for large and small Islamic banks in Southeast Asian countries; the coefficient is strongly negative and significant in several specifications at the 1 and 10 percent levels. A move from lending-based operations to other sources of income might increase the insolvency risk in Islamic banks.

Our use of the GDP as variable is intended to capture the effect of macroeconomic conditions (business cycles) on z-score. As expected, GDP growth is positive for the full sample in Southeast Asian countries and for large banks in MENA countries; the results are significant at the 1 and 5 percent levels, suggesting a business cyclical behavior of $z$-scores in these countries, except for small banks in MENA countries, where the results are significant and negative at the 1 percent level. Consumer price inflation leads to banking instability as shown by its negative coefficient which is significant at the 1 percent level for large banks in Southeast Asian countries. Nevertheless, the coefficient is positive and significant at the 1 percent level for small banks in Southeast Asian countries. The official exchange rate plays a particularly important role in financial instability as shown by its significant negative coefficient for large banks in Southeast Asian countries at the 5 percent level, except in MENA countries.

Our econometric results indicate that with a negative coefficient; an increase in LIBOR leads to a decrease in z-scores in small Islamic banks in Southeast Asian countries at the 5 percent level. In a dual system, Islamic banks' balance sheets in Southeast Asian countries are exposed to variations of rates of return linked to LIBOR despite operating on interest-free principles. For large Islamic banks in MENA countries, our result indicates that the interest rate risk is significant at the 5 percent level with a positive coefficient. Consequently, an increase in LIBOR leads to an increase in z-scores in large Islamic banks in MENA countries.

For large banks in MENA and Southeast Asian countries, a higher presence of conventional banks in a banking system has a positive impact on $\mathrm{z}$ scores at the 1 and 10 percent levels. Finally, conventional banks contribute to the overall stability of a banking system. For small banks in Southeast Asian countries, we can clearly observe the association between a presence of conventional banks and instability. The coefficients are negative in the specifications (2) and (4) at the 1 percent level. The interaction between the market share of conventional banks and the Islamic banks dummy indicates that a higher presence of conventional banks does have a significant negative impact on z-scores of large Islamic banks in Southeast Asian countries at the 5 percent level. This result could be justified by the asynchronous reactions of conventional banks which certainly affect the overall stability of a banking system by increasing systemic risk. Our results indicate that in Southeast Asian countries, large Islamic and conventional banks cannot coexist in a competitive market without crowding out effects. Finally, we find that the presence of conventional banks 
has a positive impact on the soundness of small Islamic banks in MENA countries, where the results are significant in the specifications (3) and (4) at the 1 percent level.

MENA countries recorded a substantial decrease of concentration in banking sectors during the last decade. ${ }^{18}$ Looking at the movements in rolling coefficients, we find that a higher concentration in the banking sector contributed to instability. For small banks, the impact of the Herfindahl-Hirschman index is significantly negative in Southeast Asian countries at the 1 percent level. However, as concentration later decreased, this relation finally became negative in Southeast Asian countries, suggesting that a lower concentration with stronger competition in the banking sector contributed to lower insolvency risk. This is in line with the literature on banking sector concentration and stability that finds higher concentration to be associated with lower stability (Boyd, de Nicoló and Jalal, 2006; Schaeck, Čihák and Wolfe, 2009). For large banks in Southeast Asian countries, the findings are the opposite, where a higher level of concentration increases the z-score in all specifications at the 5 and 10 percent levels. Southeast Asian countries recorded a substantial increase of banking sector concentration during the last decade ${ }^{19}$ Concentration in banking sectors is positively associated with stability in large banks. This result suggests that large banks should increase their size through mergers and acquisitions in order to achieve economies of scale and avoid failures. A higher concentration with weaker competition contributed to lower insolvency risk. This is in line with some other studies exploring the same relation in a much broader sample of banks (Carletti and Hartmann, 2003; Allen and Gale, 2004).

The governance variable tends to be strongly positive in all regressions in which it is entered, suggesting, as expected, that better governance is correlated with higher z-scores. ${ }^{20}$ Our results are significant at the 1 percent level, except for small banks in MENA countries. ${ }^{21}$ Thus, the quality of institutions as measured by the governance variable does not statistically influence the insolvency for small banks in MENA countries. Unlike studies that have found that institutions matter for financial risk (Čihák and Hesse, 2010), we find that insolvency appears impervious to the quality of the institutional environment in MENA countries. It may be that regardless of the institutional environment, the way Islamic banks are permitted to behave is driven by relatively strict Sharia law,

\footnotetext{
${ }^{18}$ Over the last two decades MENA countries, as well as much of the developing countries, have experienced a wave of liberalization of the financial sector.

${ }^{19}$ An increase in concentration can be related to the process of consolidation and restructuring. Due to the severe impact of the 1998-2001 East Asian financial crisis, the Bank Negara Malaysia forced mergers to consolidate the banking sector.

${ }^{20}$ We did not think it was necessary to lag the governance variable. It did not have an impact on the results as it does not change much year to year.

${ }^{21}$ Here Sharia Supervisory Boards were extremely cautious in their advice, and sometimes even obstructive. The quality of institutions does not matter, probably because the often higher hurdle set by Sharia law trumps the quality of local institutions in most countries (Imam and Kpodar, 2010).
} 
making the institutional environment less important than for banks in Southeast Asian countries and large banks in MENA countries.

Finally, we introduce a methodological advance using quantile regression to allow us to address the question of whether the factors that cause high fragility are systematically different from the factors that cause medium or low fragility. ${ }^{22}$ Quantile regression (table 12) can be considered superior to the previously used estimation techniques since it provides more precise estimates of the impact of the determinants of insolvency.

Given that our sample includes outliers, to control the skewness of the distribution of the z-score, the model specification includes a logarithm of the zscore which is normally distributed to smooth out higher values of the z-score. The logarithm of the z-score is most closely related to the odds of insolvency rather than the probability of insolvency itself (Strobel, 2011). Consequently, we use the logarithm of z-score, although this does not help the quantile regression fit, it helps with statistical inference (Chen, 2005). The model setup is the same as for the full sample with the same variables included in MENA and Southeast Asian countries.

The model with the z-score logged shows that in Southeast Asian countries, the median Islamic banks have a higher z-score than the conventional banks. The result is significant at the $10 \%$ level. Greater income diversity tends to increase z-scores on the weakest Islamic banks in MENA countries at the 5 $\%$ level. In Southeast Asian countries, greater income diversity tends to decrease z-scores on the weakest, median and strongest Islamic banks at the 1,5 and $10 \%$ levels. The coefficients of the credit risk and its interaction with the Islamic bank dummy in MENA countries show that credit risk has a positive effect on the median Islamic banks. The result is significant at the $5 \%$ level.

\section{CONCLUSION}

These analyses are the first to compare empirically the risk of insolvency of Islamic banks with conventional banks in MENA and Southeast Asian countries. The relative financial strength of Islamic banks is assessed empirically based on evidence covering individual Islamic and conventional banks in 16 banking systems which have a substantial presence of Islamic banking. We find that Islamic banks' z-scores are on average higher than those of conventional banks (indicating higher stability than conventional banks), except for small Islamic banks. Our results are different from those found by Čihák and Hesse (2010). These authors tried to see if market share of Islamic banks has a significant impact on the financial strength of conventional banks, arguing that

\footnotetext{
${ }^{22}$ The 50th percentile gives the median least square estimator which minimizes the median square of residuals rather than the average. In the generalized quantile regression, we estimate an equation describing a quantile other than the median. Specifically, we estimate the first quartile (25th percentile) as well as the 75th percentile.
} 
Islamic financial institutions pose a risk to the financial system that in many regards differs from those posed by the conventional financial system.

We know that Islamic banks may fail. The main contribution that we hope our work makes is to promote overall a better understanding of various biases that may affect theses failures. We observe that different causal links analyzed give us new tools for the apprehension of risks in Islamic financial institutions. Our findings demonstrate that credit risk and income diversity are the most common cause of insolvency for Islamic banks. We have shown that despite risk management, credit risk measured by the ratio of loan loss provisions to net interest income decreases the $\mathrm{z}$-score in small Islamic banks in MENA countries and large Islamic banks in Southeast Asian countries. The suggested explanation is that Islamic banks in these countries tend to have problems monitoring credit risks. Our findings demonstrate that income diversification is a cause of insolvency for small and large Islamic banks in Southeast Asian countries. A move from lending-based operations to other sources of income might increase the insolvency risk in Islamic banks in Southeast Asian countries while a move from lending-based operations to other sources of income might improve stability for large and small Islamic banks in MENA countries. Our econometric results indicate that an increase in LIBOR leads to a decrease in z-scores in small Islamic banks in Southeast Asian countries. Islamic banks in Southeast Asian countries are exposed to variations of rates of return linked to LIBOR despite operating on interest-free principles. Our results indicate that in Southeast Asian countries, large Islamic and conventional banks cannot coexist in a competitive market without crowding out effects.

Islamic banking could take several measures to improve its stability and compete with conventional counterparts. As is usually the case in social sciences, the empirical tests conducted in our analysis have limitations. This study could be completed by incorporating other variables such as stock indexes and the age or experience level of banks, certainly more difficult to quantify, but which must be taken into account. By focusing on the ownership structure (control and cash flow rights), we could examine whether this structure affects banking risks (Laeven and Levin, 2009; Sanya and Wolfe, 2010). 
ANNEX 1.

Definition of Variables

\begin{tabular}{|c|c|c|}
\hline & Variable & Description \\
\hline \multicolumn{2}{|c|}{$\begin{array}{l}\text { Dependent Variable: } \\
\text { Insolvency Risk Exposure }\end{array}$} & $\begin{array}{l}Z_{i, j, t}=\frac{\left(E(R O A A)_{i, j, t}+\frac{e q_{i, j, t}}{t a_{i, j, t}}\right)}{\sigma R O A A_{i, j, t}} \\
\text { where } E(R O A A)_{i, j, t} \text { stands for expected return on average } \\
\text { assets, } \sigma(R O A A)_{i, j, t} \text { denotes standard deviation of return } \\
\text { on assets }(R O A A)^{2} \text { as a proxy for return volatility } e q_{i, j, t} \text { is } \\
\text { bank's equity and reserves } t a_{i, j, t} \text { is bank's assets. }\end{array}$ \\
\hline \multicolumn{3}{|c|}{ Explanatory Variables: } \\
\hline \multirow[t]{8}{*}{ Bank Specific } & Log Assets & $\begin{array}{l}\text { Logarithm of the total assets of a bank (In U.S. thousand } \\
\text { dollars). }\end{array}$ \\
\hline & Assets Structure & Share of total credits in bank assets. \\
\hline & Cost to Income Ratio & Ratio of cost to income. \\
\hline & Credit Risk & Loan loss provisions to net interest income. \\
\hline & $\begin{array}{l}\text { Credit risk*Islamic Bank } \\
\text { Dummy }\end{array}$ & Interaction of credit risk and Islamic bank dummy. \\
\hline & Income Diversity & $\begin{array}{l}1 \text { - } \text { (Net interest income - Other operating income) / } \\
\text { Total operating income } \mid \text {. }\end{array}$ \\
\hline & $\begin{array}{l}\text { Income Diversity*Islamic } \\
\text { Bank Dummy }\end{array}$ & Interaction of income diversity and Islamic bank dummy. \\
\hline & Liquidity Risk & $\begin{array}{l}\text { Liquid assets as percentage of customer and short term } \\
\text { funding. }\end{array}$ \\
\hline \multirow[t]{3}{*}{ Banking Sector } & Concentration & Herfindhal-Hirschman Index. \\
\hline & $\begin{array}{l}\text { Market Share of Conven- } \\
\text { tional banks }\end{array}$ & Market share of conventional banks in a country per year. \\
\hline & $\begin{array}{l}\text { Market Share of Conven- } \\
\text { tional Banks*Islamic Bank } \\
\text { Dummy }\end{array}$ & $\begin{array}{l}\text { Interaction of market share of conventional banks and } \\
\text { Islamic bank dummy. }\end{array}$ \\
\hline \multirow[t]{4}{*}{$\begin{array}{l}\text { Macroeconomic } \\
\text { Variables }\end{array}$} & GDP Growth & $\begin{array}{l}\text { Growth rate of nominal GDP, adjusted for inflation } \\
\text { (Percent). }\end{array}$ \\
\hline & Inflation & Year-on-year change of the CPI index (Percent). \\
\hline & $\begin{array}{l}\text { London interbank offered } \\
\text { rate }\end{array}$ & $\begin{array}{l}\text { The official exchange rate refers to the exchange rate } \\
\text { determined by national authorities or the rate determined } \\
\text { in the legally sanctioned exchange market. It is calculat- } \\
\text { ed as an annual average based on monthly averages } \\
\text { (local currency units relative to the U.S. dollar), trans- } \\
\text { formed in percent. } \\
\text { 6-months LIBOR. }\end{array}$ \\
\hline & $\begin{array}{l}\text { London interbank offered } \\
\text { rate*Islamic Bank dummy }\end{array}$ & Interaction of LIBOR and Islamic bank dummy. \\
\hline Governance & Governance & $\begin{array}{l}\text { Voice and accountability, political stability, government } \\
\text { effectiveness, regulatory quality, rule of law, control of } \\
\text { corruption (2000-2008). }\end{array}$ \\
\hline Dummy Variable & Islamic Bank Dummy & Equals 1 for Islamic banks, 0 otherwise. \\
\hline \multicolumn{3}{|c|}{$\begin{array}{l}\text { Sources: All microeconomic variables are taken from BankScope database. The Z-score, the Herfindhal- } \\
\text { Hirschman Index and the market share of conventional banks are the author's calculations based on the } \\
\text { BankScope database. The macroeconomic variables are taken from the IFS (International Financial Statistics } \\
\text { published by the International Monetary Fund }(I M F) \text {. The LIBOR variable is taken from the Mortgage-X } \\
\text { LIBOR Index. The dummy variable and the interactions are the author's calculations. The governance varia- } \\
\text { ble is taken from Kaufmann, Kraay and Mastruzzi (2010). Data frequency: annual. }\end{array}$} \\
\hline
\end{tabular}




\section{REFERENCES}

Abdul-Majid, M., Saal, D. S. and Battisti, G., 2010, "Efficiency in Islamic and Conventional Banking: An International Comparison", Journal of Productivity Analysis, 34, 25-43.

Allen, F. and Gale, D., 2004, "Competition and Financial Stability", Journal of Money, Credit and Banking, 36(3), 453-480.

Ariss, R. T., 2010, "Competitive Conditions in Islamic and Conventional Banking: A Global Perspective", Review of Financial Economics, 19(3), 101-108.

Bader, M. K. I., Mohamad, S., Ariff, M. and Hassan, T., 2008, "Cost, Revenue and Profit Efficiency of Islamic versus Conventional Banks: International Evidence Using Data Envelopment Analysis", Islamic Economic Studies, 15, 23-76.

Beck, T., Demirgüç-Kunt, A. and Merrouche, O., 2010, "Islamic vs. Conventional Banking Business Model, Efficiency and Stability", Policy Research Working Paper 5546, Finance and Private Sector Development Team, World Bank, Washington, D.C., $44 \mathrm{p}$.

Berger, A., 1995, "The Relationship between Capital and Earnings in Banking", Journal of Money, Credit and Banking, 27, 432-456.

Borio, C. and Zhu, H., 2012, "Capital Regulation, Risk-Taking and Monetary Policy: A Missing Link in the Transmission Mechanism?", Journal of Financial Stability, 8(4), 236-251.

Boyd, J. H. and De Nicoló, G., 2005, "The Theory of Bank Risk Taking and Competition Revisited", The Journal of Finance, 60(3), 1329-1343.

Boyd, J. H., De Nicoló, G. and Jalal, Abu M., 2006, "Bank Risk-Taking and Competition Revisited: New Theory and New Evidence", IMF Working Paper 06/297, International Monetary Fund, Washington, D.C., 51 p.

Carletti, E. and Hartmann, P., 2002, "Competition and Stability: What's Special about Banking?", European Central Bank Working Paper 146, 50 p.

Chapra, U. and Ahmed, H., 2002, "Corporate Governance in Islamic Financial Institutions", Occasional Paper 6, Islamic Research and Training Institute, Islamic Development Bank, Jeddah.

Chen, C., 2005, "An Introduction to Quantile Regression and the QUANTREG Procedure", Statistics and Data Analysis SUGI 27, SAS Institute Inc.

Chen, C., 2007, "Robust Regression and Outlier Detection with the Robustreg Procedure", Statistics and Data Analysis SUGI 30, SAS Institute Inc.

Chong, B. and Liu, M., 2009, "Islamic Banking: Interest-Free or Interest-Based?", Pacific-Basin Finance Journal, 17(1), 125-144.

Čihák, M. and Hesse, H., 2007, "Cooperative Banks and Financial Stability", IMF Working Paper 07/2, International Monetary Fund, Monetary and Capital Markets Dept., Washington, D.C., 36 p.

Čihák, M. and Hesse, H., 2010, "Islamic Banks and Financial Stability : An Empirical Analysis", Journal of Financial Services Research, 38(2-3), 19 p. 
Dell'Ariccia, G. and Marquez, R., 2006, "Lending booms and lending standards", The Journal of Finance, 61, 2511-2546.

Delis, M.D. and Kouretas, G.P., 2011, "Interest rates and bank risk-taking", Journal of Banking and Finance, 35, 840-855.

Grais, W. and Kulathunga, A., 2006, "Capital Structure and Risk in Islamic Financial Services", Islamic Finance: The Regulatory Challenge, John Wiley and Sons, Singapore, $418 \mathrm{p}$.

Grais, W. and Pellegrini, M., 2006, "Corporate Governance and Shariah Compliance in Institutions Offering Islamic Financial Services", World Bank Policy Research Working Paper, $n^{\circ} 4054$.

Hasan, M. and Dridi, J., 2010, "The Effects of the Global Crisis on Islamic and Conventional Banks: A Comparative Study", IMF Working Paper 10/201, International Monetary Fund, Monetary and Financial Systems Dept., Washington, D.C., $47 \mathrm{p}$.

Imam, P. and Kpodar, K., 2010, "Islamic Banking: How Has it Diffused?", IMF Working Paper 10/195, International Monetary Fund, Washington, D. C., 30 p.

Kaufmann, D., Kraay, A. and Mastruzzi, M., 2010, "Governance Indicators for 20002008, The Worldwide Governance Indicators (WGI) project", World Bank Policy Research, Washington, D.C.

Koenker, R., 2005, Quantile Regression, Econometric Society Monograph,Cambridge University Press, Cambridge, $349 \mathrm{p}$.

Laeven, L. and Levine, R., 2007, "Is There a Diversification Discount in Financial Conglomerates", Journal of Financial Economics, 85(2), 331-367.

Laeven, L. and Levine, R., 2008, "Complex Ownership Structures and Corporate Valuations", Review of Financial Studies, 21(2), 579-604.

Laeven, L. and Levine, R., 2009, "Bank governance, Regulation and Risk Taking", Journal of Financial Economics, 93(2), 259-275.

Levine, R. and Zervos, S., 1998, "Stock Markets, Banks and Economic Growth", American Economic Review, 88(3), 537-558.

Levine, R., 2005, "Finance and Growth: Theory and Evidence", Handbook of Economic Growth, Volume 1, Part A, 865-934.

Maddaloni, A. and Peydró, J., 2011, "Bank Risk-Taking, Securitization, Supervision, and Low Interest Rates: Evidence from the Euro Area and U.S. Lending Standards", Review of Financial Studies, 24, 2121-2165.

Hamim, S. A. M., Naziruddin, A. and Syed, M. Al-H., 2006, "Efficiency of Islamic Banks in Malaysia: A Stochastic Frontier Approach", Journal of Economic Cooperation among Islamic Countries, 27(2), 37-70.

Rajan, R. and Zingales, L., 1998, "Financial Dependence and Growth", American Economic Review, 88(3), 559-586.

Sanya, S. and Wolfe, S., 2010, "Ownership Structure, Revenue Diversification and Insolvency Risk in European Banks", Available at Social Science Research Network (SSRN).

Schaeck, K. and Čihák, M., 2010, "Banking Competition and Capital Ratios", European Financial Management, 18, 836-866. 
Schaeck, K., Čihák, M. and Wolfe, S., 2009, "Are More Competitive Banking Systems More Stable", Journal of Money, Credit and Banking, 41(4), 711-734.

Strobel, F., 2010, "Bank Insolvency Risk and Aggregate Z-score Measures: a Caveat", Economics Bulletin, 30 (4), 2576-2578.

Strobel, F., 2011, "Bank Insolvency Risk, Z-Scores and an Improved Insolvency Probability Bound", Available at Social Science Research Network (SSRN).

Sundarajan, V. and Errico, L., 2002, "Islamic Financial Institutions and Products in the Global Financial System : Key Issues in Risk Management and Challenges Ahead", IMF Working Paper 02/192, International Monetary Fund, Monetary and Exchange Affairs Department, Washington, D.C., 27 p.

Weill, L., 2009, "Do Islamic Banks Have Greater Market Power?", Comparative Economic Studies, 53 (2), 291-306.

Yudistira, D., 2004, "Efficiency in Islamic Banking: An Empirical Analysis of Eighteen Banks", Islamic Economic Studies, 12(1), 1-19.

\section{BANQUES ISLAMIQUES ET STABILITÉ FINANCIÈRE : UNE ANALYSE COMPARÉE DES PAYS MENA ET DE L'ASIE DU SUD-EST}

Résumé - L'objectif de cet article est d'évaluer la stabilité financière des banques islamiques et des banques conventionnelles. Pour mesurer la stabilité financière, nous calculons sur la période 2000-2008 le z-score pour 16 pays où les deux types de banques coexistent. A cette fin, nous utilisons un modèle d'estimation robuste et quantile afin de comparer les causes de l'insolvabilité des banques islamiques et conventionnelles dans les pays du Moyen Orient et de l'Afrique du Nord et celles du Sud-Est asiatique. Finalement, en utilisant différents facteurs et une analyse comparative entre les régions, cet article tente d'offrir une extension à l'étude de Cihák et Hesse (2010).

Mots-clés : BANQUE ISLAMIQUE, STABILITE FINANCIERE, Z-SCORE, MENA, ASIE DU SUD-EST 